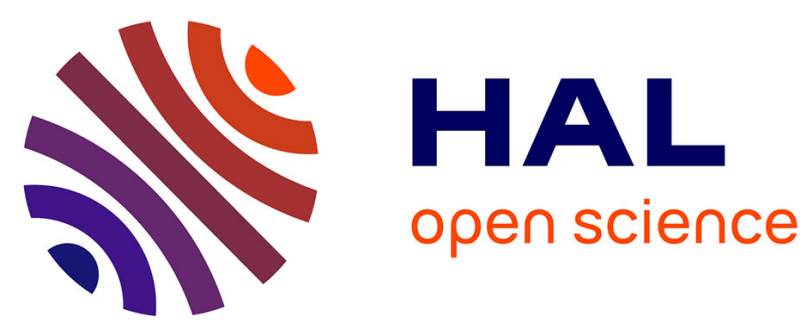

\title{
In vivo imaging of inflammasome activation reveals a subcapsular macrophage burst response that mobilizes innate and adaptive immunity
}

Pervinder Sagoo, Zacarias Garcia, Beatrice Breart, Fabrice Lemaître, David Michonneau, Matthew L Albert, Yves Levy, Philippe Bousso

\section{To cite this version:}

Pervinder Sagoo, Zacarias Garcia, Beatrice Breart, Fabrice Lemaître, David Michonneau, et al.. In vivo imaging of inflammasome activation reveals a subcapsular macrophage burst response that mobilizes innate and adaptive immunity. Nature Medicine, 2015, 22 (1), pp.64-71. 10.1038/nm.4016 . pasteur-01253201

HAL Id: pasteur-01253201

https://hal-pasteur.archives-ouvertes.fr/pasteur-01253201

Submitted on 8 Jan 2016

HAL is a multi-disciplinary open access archive for the deposit and dissemination of scientific research documents, whether they are published or not. The documents may come from teaching and research institutions in France or abroad, or from public or private research centers.
L'archive ouverte pluridisciplinaire HAL, est destinée au dépôt et à la diffusion de documents scientifiques de niveau recherche, publiés ou non, émanant des établissements d'enseignement et de recherche français ou étrangers, des laboratoires publics ou privés.

\section{(ㅇ)(1) $\$$}

Distributed under a Creative Commons Attribution - NonCommercial - NoDerivatives| 4.0 


\title{
In vivo imaging of inflammasome activation reveals a subcapsular macrophage burst response that mobilizes innate and adaptive immunity
}

\author{
Pervinder Sagoo $^{1,2}$, Zacarias Garcia ${ }^{1,2}$, Beatrice Breart ${ }^{1,2}$, Fabrice Lemaître ${ }^{1,2}$, \\ David Michonneau ${ }^{1,2}$, Matthew L. Albert ${ }^{3,4}$, Yves Levy ${ }^{5,6,7,8}$ \\ and Philippe Bousso ${ }^{1,2,3}$
}

${ }^{1}$ Dynamics of Immune Responses Unit, Institut Pasteur, Paris, France.

${ }^{2}$ Institut National de la Santé et de la Recherche Médicale U668, rue du Dr Roux, Paris, France.

${ }^{3}$ Laboratory of Dendritic Cells Immunobiology, Institut Pasteur, Paris, France;

${ }^{4}$ Institut National de la Santé et de la Recherche Médicale U818, Paris, France.

${ }^{5}$ Vaccine Research Institute, Créteil, France.

${ }^{6}$ Institut National de la Santé et de la Recherche Médicale U955, Créteil, France

${ }^{7}$ Faculté de Médecine, Université Paris Est, Créteil, France

${ }^{8}$ Service d'immunologie clinique et maladies infectieuses, AP-HP, Hôpital H. Mondor-A.

Chenevier, Créteil, France

Correspondence to: philippe.bousso@pasteur.fr 
The inflammasome pathway is activated in response to a variety of pathogens and plays an important role in shaping adaptive immunity. Yet, the spatiotemporal orchestration of inflammasome activation in vivo and the mechanisms by which it promotes an effective immune response remain to be fully understood. Using an in vivo reporter to visualize inflammasome assembly, we establish the distribution, kinetics and propagation of the inflammasome response to a local viral infection. We show that Modified Vaccinia Ankara virus induces inflammasome activation in subcapsular sinus (SCS) macrophages, immediately followed by cell death and release of extracellular ASC specks. This transient inflammasome signaling in the lymph node generates a robust influx of inflammatory cells, and importantly, mobilizes $T$ cells from the circulation to increase the magnitude of $\mathrm{T}$ cell responses. We propose that, in response to infection, SCS macrophages deliver a burst response of inflammasome activity and cell death that translates into the broadening of $T$ cell responses, identifying an important advantage of inflammasome driven vaccination strategies. 


\section{Introduction}

Innate immune sensing of pathogens or damage is characterized by activation of membrane and cytosolic pattern recognition receptors (PRR) including Toll-like receptors (TLRs), retinoic acid inducible gene I-like helicase receptors (RLRs), cytosolic DNA sensors, and nucleotide oligomerization domain-like receptors (NLRs) ${ }^{1}$. The inflammasome forms part of this first line response, mediated primarily by phagocytic antigen-presenting cells such as macrophages and dendritic cells, and refers to a hetero-multimeric protein scaffold which is assembled upon activation of cytosolic PRRs ${ }^{2}$. Multiple inflammasome complexes exist, each being triggered by several cytosolic sensor protein families with specificities for distinct pattern-recognition motifs ${ }^{2}$. Once activated, many of these sensors associate with a common adaptor protein known as ASC (apoptosis-like speck protein with a CARD domain, Pycard), which promotes recruitment and proximity-induced autocatalytic maturation of caspase- $1^{3}$. These processes occur through prion-like polymerization of ASC into filaments and ultimately result in the formation of micrometer-sized structures referred to as ASC specks ${ }^{4}$. Inflammatory effector mechanisms unleashed by active caspase-1 include the proteolytic processing and release of bioactive interleukins $1 \beta \quad$ (IL-1 $\beta$, IL-18), and induction of cellular pyroptotic death ${ }^{4,5}$. In vitro, ASC speck formation can coincide with cell death and cytokine release ${ }^{6,7}$ but the relationship between inflammasome activation in vivo and induction of cell death remains to be documented. Recent studies have provided evidence that ASC specks can exist as active extracellular structures, raising interest in the fate and dynamics of ASC specks after cell death ${ }^{8,9}$. Despite our increasing knowledge of effector mechanisms induced by the inflammasome response, we still have a limited understanding of how inflammasome activation unfolds in vivo. In particular, the location and timing of inflammasome activity in tissue remain unclear, as do the immediate effects of cellular inflammasome activity. While several fluorescent probes have been developed to study 
inflammasome activation in vitro ${ }^{10-12}$, their use for in vivo applications has remained challenging, lacking translation to single cell resolution at the organ level ${ }^{10,12,13}$.

Another aspect of inflammasome biology is to understand how its activity regulates innate and adaptive immunity. Pro-inflammatory IL-1 and IL-18 have been shown to play roles in both local and peripheral recruitment and priming of the immune response ${ }^{14-16}$, and absence of inflammasome activity has been associated with poorer pathogen clearance and increased disease severity ${ }^{17-21}$. Mechanisms that link the inflammasome pathway to $\mathrm{T}$ cell responses include danger associated molecular pattern (DAMP)-mediated dendritic cell (DC) activation $^{22}$, effects of inflammatory cytokines on $\mathrm{T}$ cell polarization ${ }^{23}$ and activation, or noncognate induction of IFN- $\gamma$ in memory $\mathrm{T}$ cells ${ }^{16,24}$. In this respect, understanding the spatiotemporal dynamics of inflammasome activation may help clarify the downstream effects on the $\mathrm{T}$ cell response. Here, we establish an in vivo reporter system to visualize inflammasome activation in real-time and dissect its influence on the immune response following a local viral infection. 


\section{Results}

\section{MVA induces inflammasome activation in macrophages}

To examine local viral infection as an inflammasome trigger, we selected Modified Vaccinia Ankara virus (MVA), a replication deficient group I dsDNA poxvirus used in clinical vaccination programmes, with strong immunogenicity through activation of endosomal TLRs and cytosolic MDA-5, AIM2 and NLRP3 ${ }^{25-27}$. As such, it delivers a temporally defined stimulus for inflammasome activation, allowing a model for direct monitoring of downstream cellular events of infection. Using both wild type (WT) and Caspase-1 (Casp1 $1^{-/-} /$Casp $\left.^{-1} / 11^{-/-}\right)$ deficient mouse strains, we confirmed that MVA infection induced inflammasome activity in bone-marrow derived macrophages (BMDM), by detection of caspase-1 activity (Fig. 1a and Supplementary Fig. 1a) and IL-1 $\beta$

Fig. 1b . Addition of Z-YVAD-FMK caspase- 1 specific inhibitor prevented IL-1 $\beta$ release induced by adenosine triphosphate (ATP) treatment of LPS-primed BMDM and by MVA-infected BMDM, demonstrating that IL-1 $\beta$ release depended on the conventional inflammasome pathway ${ }^{28,29}$.

To detect inflammasome activation in real-time, we stably transduced BMDM to express a fusion protein of ASC and green fluorescent protein (ASC-GFP), as a read-out for inflammasome assembly. As anticipated, MVA infection induced formation of ASC 'speck' inflammasomes in vitro (Fig. 1c-d), which could be visualised by redistribution of ASC-GFP protein as it oligomerizes to form a single macromolecular complex ${ }^{30}$ (Supplementary movie 1). Concomitant with inflammasome activity, MVA infection also induced caspase-1 dependent macrophage death (Fig. 1e). In vitro ASC-overexpression did not alter cellular levels of caspase-1 activity, IL-1 $\beta$ release, nor the kinetics or amount of cell death (Supplementary Fig. 1b-f), which corresponds with a recent report highlighting the critical role of the levels of upstream inflammasome sensor proteins in determining the rate of speck 
formation $^{4}$. In vivo after local viral infection (subcutaneous (s.c.) footpad injection) with MVA, we found that virus primarily targeted macrophages within the subcapsular (SCS-M) and medullary (Med-M) regions of the draining lymph node, consistent with previous reports of their role in sequestering lymph borne pathogens ${ }^{31-33}$ (Supplementary Fig. 2). MVA infection resulted in rapid and specific loss of lymph node macrophages, most notably CD169 ${ }^{+}$F4/80 ${ }^{-}$SCS-M (Fig. 1f-g). These findings suggest that inflammasome signaling and pyroptotic death may be an important component of the immune response to MVA.

\section{SCS macrophages initiate the inflammasome response to MVA}

Detection of inflammasome activity in vivo at single cell resolution has so far been restricted to static imaging on fixed tissue sections ${ }^{34}$, limiting our understanding of the identity, dynamics and fate of cells undergoing inflammasome activation. This is in part due to the lack of mouse models to detect inflammasome activation and in part due to the inherently transient nature of cell signaling which may ultimately progress to cellular demise.

To circumvent this limitation, we developed a strategy for dynamic two-photon imaging of inflammasome assembly by in vivo detection of ASC speck formation. Mixed bone marrow chimeric mice were generated by transfer of cycling bone marrow-derived haematopoietic stem cells (BM-HSC), retrovirally transduced to express ASC-GFP fusion protein. ASC-GFP-expressing BM-HSC, derived from Rag $^{-/-}$mice, were mixed with either WT or caspase-1-deficient BM-HSC, and used to reconstitute recipient mice (Fig. 2a). This strategy served to restrict reporter expression to the myeloid compartment, and limit caspase1-dependent functional inflammasome signaling to those cells expressing the reporter. Within lymph nodes of chimeric mice, ASC-GFP was expressed by myeloid cells, with a normal distribution throughout the lymph node volume (Supplementary Fig. 3a-b). Intravital imaging of chimeric mice revealed, in particular, ASC-GFP expression by cells localized to 
the subcapsular sinus region of resting lymph nodes (Fig. 2b). These cells formed a dense network at the lymph node periphery, displayed a macrophage-like morphology and were stained following in vivo injection of a CD169-specific antibody (Fig. 2c), indicating myeloid-derived reconstitution of lymph node subcapsular sinus macrophages, as previously described $^{35}$.

When ASC-GFP chimeric mice were injected s.c. with MVA, we detected ASC speck formation occurring primarily within these macrophages in draining lymph nodes (Fig. 2d and Supplementary movie 2). Of note, we did not observe ASC speck formation in control mice injected with PBS (Fig. 2e and Supplementary movie 3). Single-cell analysis of the redistribution of ASC-GFP protein leading to speck assembly, revealed a rapid and dynamic cellular process (Fig. 2f-g), with the majority of cells undergoing complete ASC oligomerization within 10 minutes (mode 2.8; median 6; range 2-99 mins) (Fig. 3a and Supplementary Fig. 3c).

We next quantified ASC speck formation occurring during the first 12 hours following local MVA injection and identified an acute inflammasome response, detectable within one hour following infection and peaking over the subsequent 2 hours (Fig. 3c). For these analyses, we counted ASC specks forming during the imaging (forming ASC specks) as well as ASC specks already formed (formed ASC specks) at the time of imaging. Inflammasome activation then steadily declined until neither active speck formation, nor formed specks were detected at 12 hours. The number of ASC specks detected following infection corresponded with the kinetics of SCS-M loss observed by histological and lymph node cell suspension analysis methods shown earlier (Fig. 1f-g). Thus the steady decline in the numbers of specks detected post-infection, likely reflects the loss of SCS-macrophages. Throughout the imaging period, ASC specks were not detected in lymph nodes of PBS-injected controls, nor within deeper regions of the lymph node accessible by two-photon imaging following MVA 
injection (Supplementary movie 4). Further analysis of the relative spatial arrangement of specks in the lymph node revealed that the majority were localised to within $30 \mu \mathrm{m}$ of the subcapsular region, indicated by the presence of capsular collagen fibers (Fig. 3c and Supplementary Fig. 3d-e). When compiled, these data reveal a rapid triggering of ASC speck formation, generating a transient and spatially confined wave of inflammasome activity spanning the first 7 hours following infection.

\section{Cell death and release of extracellular ASC specks in vivo}

Pyroptosis is a caspase- 1 dependent form of cell death ${ }^{36}$, which unlike other modes of cell death, is an inflammatory event associated with cell swelling, membrane lysis and nuclear condensation $^{37}$. Using time-lapse microscopy of BMDM we examined infection (MVA-GFP), inflammasome activation (ASC-mcherry) and cell death (DNA binding SytoxBlue) events in parallel (Fig. 4a and Supplementary movie 5). Dynamic analysis of virally encoded GFP and nuclear stain intensities revealed that ASC speck formation in BMDM was closely followed by loss of cell integrity and cellular demise (Fig. 4b). This rapid process of cell death occurred within minutes following inflammasome assembly (mode 3 min; median 7 min; range 0-40 $\mathrm{min}$ ) and was largely intrinsic to cell infection, with no appreciable cell-tocell transactivation of the inflammasome response to infection (Supplementary Fig. 4a-b). We next examined the process of ASC speck formation and cell death by in vivo labelling of lymph nodes with the cell permeable nuclear DNA binding dye Hoechst 33342, enabling detection of cell death events by tracking nuclear condensation. Intravital imaging revealed that macrophages undergoing inflammasome activation died rapidly after speck formation,

progressing firstly to nuclear condensation and then disappearance of the nucleus (Fig. 4c, Supplementary Fig. 4c). 
While examining in vivo Hoechst labelling of draining lymph nodes, we noted that the vast majority of formed specks we detected did not have any associated nuclei, suggesting they were remnants of cells after inflammasome activation. To more firmly establish this hypothesis, we injected a fluorescently conjugated ASC-specific antibody into MVA-treated ASC-GFP chimeric mice. Formed specks were counterstained with the ASC antibody, confirming their extracellular nature (Fig. 4d). Intravital tracking of formed specks over several hours revealed that many specks are long-lived entities, persisting over 4 hours within the lymph node microenvironment (Fig. 4e). Moreover, we observed that specks generated in vivo either remained as long-lasting structures, gradually diminished in size and eventually disappeared, suddenly disappeared, or fragmented into multiple specks (Fig. 4e,f and Supplementary movie 6). In addition, on several occasions we also observed the acquisition of specks by other cells within the lymph node (Supplementary movie 7). These in vivo observations are consistent with two recent reports describing the ability of macrophages to phagocytize formed specks in vitro, with the potential to precipitate inflammasome activation within the engulfing cell ${ }^{8,9}$.

In vivo analysis of inflammasome activity also revealed a phenomenon whereby ASC speck formation and subsequent cell death resulted in a rapid localized recruitment and clustering of resident cells towards newly generated specks (Fig. 4g and Supplementary movie 8). Such recruitment occurred within minutes following speck formation and cell death, and could be detected using either Hoechst staining or by tracking myeloid cells $\left(\mathrm{GFP}^{+}\right)$in ASC-GFP chimeras (Supplementary movie 8). In summary, our results describe the rapid and localized initiation of inflammasome activation in the lymph node on viral infection results in macrophage death, persistence of extracellular ASC specks and local cell recruitment. 


\section{Inflammasome signaling generates a robust influx of inflammatory cells}

Our in vivo analysis revealed that inflammasome activation induced by MVA is restricted in both time and space within the lymph node. Therefore, we next assessed how inflammasome signaling translated to activation of the innate and adaptive immune response by performing whole lymph node screening of chemokine mediators expressed early (4 hours) after local viral infection (Fig. 5a). We identified a rapid production of chemokines induced by MVA, many of which were reduced in the absence of caspase-1 or IL-1 receptor signaling (CCL11, CCL22, CXCL2, CXCL10, CCL19). This shows that a transient wave of inflammasome activation elicited by MVA is a potent modulator of the chemokine release in draining lymph nodes. Consistent with these data and previous reports on chemokine release induced by $\mathrm{MVA}^{25,38}$, this was associated with rapid and robust infiltration of neutrophils, monocytes and NK cells to the lymph node. Innate immune cell recruitment was substantially reduced in the absence of caspase-1 or IL-1R1 signaling (Fig. 5b-d, supplementary Fig. 5).

To identify which cellular compartment was responsible for responding to inflammasome-derived IL-1 signals, BM chimeric mice were generated in which only hematopoietic cells $\left(\mathrm{WT} \mathrm{BM}>I l 1 r 1^{-/-}\right.$), or only non-hematopoietically derived cells $\left(I l l r 1^{-/-}\right.$ $\mathrm{BM}>\mathrm{WT}$ ) expressed IL-1R1. Infection-induced recruitment of neutrophils and monocytes was abolished in the absence of haematopoietic cellular sensing of IL-1, and was partially diminished by non-hematopoietic cell expression of IL-1R (Fig. 5e,f). This indicates that cells of predominantly hematopoietic origin relay IL-1 signals in the lymph node to promote infiltration of innate cells to the draining lymph node.

\section{Inflammasome signals broaden the composition of $\mathrm{T}$ cell responses}

To assess the contribution of inflammasome activation towards the adaptive immune response, we focused on the generation of the antigen-specific $\mathrm{CD} 8^{+} \mathrm{T}$ cell response directed 
against the immunodominant vaccinia viral peptide (VVp) B8R 20-27 expressed by MVA (Fig. 6a). Lack of caspase-1 activity or IL-1R1 expression resulted in moderately lower percentages of antigen-specific T cells induced by MVA infection (Fig. 6b). However, both caspase-1 and IL-1R1 deficient mice had reduced absolute numbers of VVp-specific cells (Fig. 6c).

We hypothesized that an overall increase in leukocyte infiltration induced by inflammasome activity may potentially lead to more naïve $\mathrm{T}$ cells being recruited to the draining lymph node for priming and activation. Indeed, functional inflammasome and IL-1R signaling promoted the overall accumulation of $\mathrm{CD}^{+} \mathrm{T}$ cells in the draining lymph node as early as 18 hours following MVA injection (Fig. 6d,e). To test whether inflammasome activation favors the recruitment of $\mathrm{T}$ cells, which were not initially present in the lymph node, T cells were transferred into WT, caspase-1- or IL-1R1-deficient recipients, followed immediately by local MVA infection (Fig. 6f) so that the majority of transferred T cells were present in the circulation at the time of infection. A combination of $\mathrm{T}$ cells were transferred that could be subsequently re-traced in vivo, each at a different ratio to provide minor circulating $\mathrm{T}$ cell populations, in order to simulate an in vivo limiting dilution assay. The specific contribution of transferred $\mathrm{T}$ cells in the bulk of the VVp specific $\mathrm{T}$ cell pool at the peak of the response was analysed in order to evaluate the mobilization of $\mathrm{T}$ cells that were not initially present in the lymph node. In WT mice, a large fraction of the VVp response was derived from the transferred $\mathrm{T}$ cell populations. However, this contribution was largely reduced in caspase-1 deficient and even more so in IL-1R deficient mice (Fig. $\mathbf{6 g , h}$ ). In particular, the $\mathrm{CFP}^{+} \mathrm{T}$ cells (that were transferred in the lowest numbers) were virtually absent from the VVp-specific response in caspase-1 and IL-1R deficient mice. We confirmed that this effect was attributed to the defective early recruitment of adoptively transferred $\mathrm{T}$ cells from the circulation to infection draining lymph nodes, in the absence of inflammasome 
signaling (Supplementary Fig. 6). As expected, delaying MVA injection until 48 hours after $\mathrm{T}$ cell transfer, allowing time for transferred $\mathrm{T}$ cells to equilibrate within lymphoid organs, resulted in the overall $\mathrm{T}$ cell response being less dependent upon early inflammasome-driven $\mathrm{T}$ cell recruitment, with transferred $\mathrm{T}$ cells making similar contributions towards the overall VVp specific T cell response in WT mice and mice with disrupted inflammasome signaling (Supplementary Fig. 6b).

In line with these findings, blocking $\mathrm{T}$ cell trafficking to the draining lymph node during MVA infection in WT mice, using a CD62L-specific blocking antibody, resulted in a significant reduction in the number of VVp specific T cells (Supplementary Fig. 7a,b). Furthermore, analysis of IFN- $\gamma \mathrm{T}$ cell responses to other $\mathrm{H}-2 \mathrm{~K}^{\mathrm{b}}$ restricted peptide epitopes for MVA (A3L, K3L, A19L, A8R), showed that blocking T cell recruitment also reduced the diversity of the $\mathrm{T}$ cell response (Supplementary Fig. 7c,d) further highlighting the importance of $\mathrm{T}$ cell trafficking to the draining lymph node for optimal responses. These data indicate that inflammasome activation increases the magnitude and broadens the potential repertoire of the $\mathrm{T}$ cell response by mobilizing antigen-specific $\mathrm{T}$ cell clones from the periphery. 


\section{Discussion}

Secondary lymphoid organs are key locations for initiation of innate host defence and priming of the adaptive response. Here we provide evidence of a host immune circuit linking activation of these two arms of the response to deliver effective immunity, which is coordinated through inflammasome signaling initiated by subcapsular macrophage sensing of infection.

Methods to detect inflammasome activation in situ have so far been very limited, relying primarily on tissue histological staining for speck protein components, active caspase1 or mature IL-1 $\beta^{8,39-41}$. This task is further complicated by the close link between inflammasome activation and cell death, and the inherent difficulty in detecting such transient events $^{42}$. Our approach circumvents these limitations by probing the inflammasome in realtime and in single cells using intravital imaging. A potential caveat of our strategy may be that ASC overexpression alters the timing of inflammasome activation. However, this was not observed in vitro, and furthermore, the kinetics of the immune response to MVA and SCS macrophage death in our chimeras was similar to that observed in wild-type mice, suggesting that overexpression of the probe has no obvious effects on the observed response. With the ability to detect the cell type, location and timing of inflammasome pathway, we provide the first glimpse into the dynamics of inflammasome activation in the context of lymph borne virus. As recent in vitro studies have demonstrated that the kinetics of caspase-1 activation are

essentially the same irrespective of the intensity or types of stimulus ${ }^{4,43}$, our results may be directly relevant to other types of pathogenic challenges in vivo.

A recent study by Kastenmuller et al. has indicated a strong role for the inflammasome pathway in innate immune cell activation and recruitment in response to lymph borne pathogens, highlighting an important role of SCS macrophages in the context of IL-18 and IL- 
$1 \beta$ release ${ }^{16}$. In addition, this report and our previous work have shown that SCS depletion alter neutrophils and NK cell recruitment in the draining lymph node upon viral challenge, respectively ${ }^{16,33}$. The present results extend these findings by directly demonstrating that macrophages in the subcapsular area of the lymph node rapidly undergo inflammasome activation, which is immediately followed by pyroptotic cell death. Although our data support the idea that SCS macrophages are the major population undergoing inflammasome activation during the early phase of the response, it is likely that other cell types, such as inflammatory monocytes or neutrophils, may also contribute towards the inflammasome response during later stages of infection ${ }^{44,45}$. Our results provide in vivo support for the idea that the inflammasome acts as a digital switch, whereby pyroptosis coincides with a burst-release of pro-inflammatory cytokines, which are rapidly processed by concomitant caspase-1 activation, a phenomenon recently described in vitro ${ }^{42,43}$. Thus, in vivo, inflammasome signaling by subcapsular sinus macrophages likely delivers a concentrated and localized inflammatory stimulus to surrounding cells, defining a discrete anatomical compartmentalization of the inflammasome response.

Extracellular ASC specks are active when injected in vivo ${ }^{8,9}$, but their generation in situ has not previously been characterized. We now directly demonstrate that most ASC specks persist for several hours as extracellular structures before showing signs of degradation. Detection of speck fragmentation and engulfment in vivo, suggests these events may provide potential mechanisms of amplifying the inflammasome response, and warrant further examination of the functional relevance of these phenomena in contributing towards the immune response.

One potential consequence of lymph borne infection is the alteration of the SCS macrophage layer. Neutrophil or dendritic cell infiltration can contribute to disrupt and/or destroy this layer ${ }^{46,47}$. Our results establish pyroptosis as an additional, direct mechanism 
accounting for the clearance of CD169 ${ }^{+}$SCS macrophages observed during infection ${ }^{47}$. Local recruitment of myeloid cells triggered by inflammasome activation and pyroptosis is reminiscent of neutrophil clustering previously observed around dying cells ${ }^{46,48,49}$. While this immediate response is likely due to the release of local mediators upon cell death such as extracellular ATP or other DAMPs ${ }^{50-52}$, our results suggest that subsequent IL-1 sensing by hematopoietic cells largely amplifies the initial inflammasome signals. This second level of signal propagation resulting in chemokine production and cellular recruitment to the draining lymph node may therefore work in concert with other mechanisms of lymph node remodelling induced by infection, such as vascular and lymph angiogenesis leading to increased organ volume ${ }^{53}$.

Emerging data is highlighting the importance of inflammasome regulation of the adaptive $\mathrm{T}$ cell response, in the context of vaccine adjuvanticity, infection and autoimmune disorders $^{54-56}$. In several studies, lack of inflammasome activity has been shown to result in a poorer $\mathrm{T}$ cell response to infection ${ }^{19,57,58}$. Triggering of the inflammasome pathway may increase the quality of antigen-presentation or act on $\mathrm{T}$ cell differentiation and expansion through the direct action of IL- $1^{24,59,60}$. Here, we have uncovered an additional mechanism whereby inflammasome activation enhances the magnitude and breadth of composition of $\mathrm{T}$ cell responses by promoting recruitment of $\mathrm{T}$ cells from the periphery. Vaccine vectors such as MVA that engage the inflammasome pathway may therefore be advantageous to mobilize a maximal number of antigen-specific $\mathrm{T}$ cell clones in the draining lymph node.

In summary, our results reveal an immune circuit operating in lymph nodes in which a spatially compartmentalized wave of inflammasome activation translates into the massive recruitment of innate and adaptive immune cells that ultimately maximises $\mathrm{T}$ cell responses. Our novel approach to probe inflammasome activity in vivo will allow dissecting the contribution of this pathway during vaccination, infections or inflammatory diseases. 


\section{Acknowledgements}

We wish to thank O. Schwartz, N. Manel and members of the Bousso laboratory for critical review of the manuscript, A. Cumano (Institut Pasteur) for helpful discussions in generating BM-Chimeric mice, and the support of the Centre d'Immunologie Humaine (Institut Pasteur). This work was supported by Institut Pasteur (P.B.), Institut National de la Santé et de la Recherche Médicale (P.B.), the Vaccine Research Institute (P.B.), Fondation pour la Recherche Médicale (P.B.) and a Starting Grant from the European Research Council (P.B.).

\section{Author contributions}

P.B. and P.S. designed the experiments, analyzed the data and wrote the manuscript. P.S. and B.B. conducted the experiments with essential support from Z.G., F.L and D.M. M.L.A and Y.L. provided scientific input and critical reagents for the experimental work.

\section{Competing financial interests}

The authors declare no competing financial interest. 


\section{References}

1. Monie, T.P., Moncrieffe, M.C. \& Gay, N.J. Structure and regulation of cytoplasmic adapter proteins involved in innate immune signaling. Immunological reviews $\mathbf{2 2 7}$ 161-175 (2009).

2. Franchi, L., Munoz-Planillo, R. \& Nunez, G. Sensing and reacting to microbes through the inflammasomes. Nature immunology 13, 325-332 (2012).

3. Bryan, N.B., Dorfleutner, A., Rojanasakul, Y. \& Stehlik, C. Activation of inflammasomes requires intracellular redistribution of the apoptotic speck-like protein containing a caspase recruitment domain. J Immunol 182, 3173-3182 (2009).

4. Lu, A., et al. Unified polymerization mechanism for the assembly of ASC-dependent inflammasomes. Cell 156, 1193-1206 (2014).

5. Fernandes-Alnemri, T., et al. The pyroptosome: a supramolecular assembly of ASC dimers mediating inflammatory cell death via caspase-1 activation. Cell death and differentiation 14, 1590-1604 (2007).

6. Stutz, A., Horvath, G.L., Monks, B.G. \& Latz, E. ASC speck formation as a readout for inflammasome activation. Methods in molecular biology 1040, 91-101 (2013).

7. Mariathasan, S., et al. Differential activation of the inflammasome by caspase-1 adaptors ASC and Ipaf. Nature 430, 213-218 (2004).

8. Franklin, B.S., et al. The adaptor ASC has extracellular and 'prionoid' activities that propagate inflammation. Nature immunology 15, 727-737 (2014).

9. Baroja-Mazo, A., et al. The NLRP3 inflammasome is released as a particulate danger signal that amplifies the inflammatory response. Nature immunology $\mathbf{1 5}, \mathbf{7 3 8 - 7 4 8}$ (2014).

10. Compan, V., et al. A genetically encoded IL-1beta bioluminescence resonance energy transfer sensor to monitor inflammasome activity. J Immunol 189, 2131-2137 (2012).

11. Compan, V., et al. Cell volume regulation modulates NLRP3 inflammasome activation. Immunity 37, 487-500 (2012).

12. Kindermann, M., et al. Selective and sensitive monitoring of caspase-1 activity by a novel bioluminescent activity-based probe. Chemistry \& biology 17, 999-1007 (2010).

13. Bartok, E., et al. iGLuc: a luciferase-based inflammasome and protease activity reporter. Nature methods 10, 147-154 (2013).

14. Dinarello, C.A. Immunological and inflammatory functions of the interleukin-1 family. Annual review of immunology 27, 519-550 (2009).

15. Rider, P., et al. IL-1alpha and IL-1beta recruit different myeloid cells and promote different stages of sterile inflammation. J Immunol 187, 4835-4843 (2011).

16. Kastenmuller, W., Torabi-Parizi, P., Subramanian, N., Lammermann, T. \& Germain, R.N. A spatially-organized multicellular innate immune response in lymph nodes limits systemic pathogen spread. Cell 150, 1235-1248 (2012).

17. Rowe, S.J., Allen, L., Ridger, V.C., Hellewell, P.G. \& Whyte, M.K. Caspase-1deficient mice have delayed neutrophil apoptosis and a prolonged inflammatory response to lipopolysaccharide-induced acute lung injury. J Immunol 169, 6401-6407 (2002).

18. Gross, O., et al. Syk kinase signalling couples to the Nlrp3 inflammasome for antifungal host defence. Nature 459, 433-436 (2009). 
19. Ichinohe, T., Lee, H.K., Ogura, Y., Flavell, R. \& Iwasaki, A. Inflammasome recognition of influenza virus is essential for adaptive immune responses. The Journal of experimental medicine 206, 79-87 (2009).

20. Shio, M.T., et al. Malarial hemozoin activates the NLRP3 inflammasome through Lyn and Syk kinases. PLoS pathogens 5, e1000559 (2009).

21. Lima-Junior, D.S., et al. Inflammasome-derived IL-1beta production induces nitric oxide-mediated resistance to Leishmania. Nature medicine 19, 909-915 (2013).

22. Ghiringhelli, F., et al. Activation of the NLRP3 inflammasome in dendritic cells induces IL-1beta-dependent adaptive immunity against tumors. Nature medicine 15, 1170-1178 (2009).

23. Chung, Y., et al. Critical regulation of early Th17 cell differentiation by interleukin-1 signaling. Immunity 30, 576-587 (2009).

24. Ben-Sasson, S.Z., et al. IL-1 enhances expansion, effector function, tissue localization, and memory response of antigen-specific CD8 T cells. The Journal of experimental medicine 210, 491-502 (2013).

25. Delaloye, J., et al. Innate immune sensing of modified vaccinia virus Ankara (MVA) is mediated by TLR2-TLR6, MDA-5 and the NALP3 inflammasome. PLoS pathogens 5, e1000480 (2009).

26. Waibler, Z., et al. Modified vaccinia virus Ankara induces Toll-like receptorindependent type I interferon responses. Journal of virology 81, 12102-12110 (2007).

27. Hornung, V., et al. AIM2 recognizes cytosolic dsDNA and forms a caspase-1activating inflammasome with ASC. Nature 458, 514-518 (2009).

28. Gross, O., Thomas, C.J., Guarda, G. \& Tschopp, J. The inflammasome: an integrated view. Immunological reviews 243, 136-151 (2011).

29. Gross, O. Measuring the inflammasome. Methods in molecular biology 844, 199-222 (2012).

30. Iwasaki, A. A virological view of innate immune recognition. Annual review of microbiology 66, 177-196 (2012).

31. Junt, T., et al. Subcapsular sinus macrophages in lymph nodes clear lymph-borne viruses and present them to antiviral B cells. Nature 450, 110-114 (2007).

32. Kastenmuller, W., et al. Peripheral prepositioning and local CXCL9 chemokinemediated guidance orchestrate rapid memory CD8 $+\mathrm{T}$ cell responses in the lymph node. Immunity 38, 502-513 (2013).

33. Garcia, Z., et al. Subcapsular sinus macrophages promote NK cell accumulation and activation in response to lymph-borne viral particles. Blood 120, 4744-4750 (2012).

34. Balci-Peynircioglu, B., et al. Expression of ASC in renal tissues of familial mediterranean fever patients with amyloidosis: postulating a role for ASC in AA type amyloid deposition. Exp Biol Med (Maywood) 233, 1324-1333 (2008).

35. Phan, T.G., Green, J.A., Gray, E.E., Xu, Y. \& Cyster, J.G. Immune complex relay by subcapsular sinus macrophages and noncognate B cells drives antibody affinity maturation. Nature immunology 10, 786-793 (2009).

36. Miao, E.A., Rajan, J.V. \& Aderem, A. Caspase-1-induced pyroptotic cell death. Immunological reviews 243, 206-214 (2011).

37. Fernandes-Alnemri, T. \& Alnemri, E.S. Assembly, purification, and assay of the activity of the ASC pyroptosome. Methods in enzymology 442, 251-270 (2008).

38. Lehmann, M.H., et al. Modified vaccinia virus ankara triggers chemotaxis of monocytes and early respiratory immigration of leukocytes by induction of CCL2 expression. Journal of virology 83, 2540-2552 (2009).

39. Dvoriantchikova, G., et al. Genetic ablation of Pannexin1 protects retinal neurons from ischemic injury. PloS one 7, e31991 (2012). 
40. Mezzaroma, E., et al. The inflammasome promotes adverse cardiac remodeling following acute myocardial infarction in the mouse. Proceedings of the National Academy of Sciences of the United States of America 108, 19725-19730 (2011).

41. Tran, H.B., et al. Immunolocalization of NLRP3 Inflammasome in Normal Murine Airway Epithelium and Changes following Induction of Ovalbumin-Induced Airway Inflammation. Journal of allergy 2012, 819176 (2012).

42. Shirasaki, Y., et al. Real-time single-cell imaging of protein secretion. Scientific reports 4, 4736 (2014).

43. Liu, T., et al. Single-Cell Imaging of Caspase-1 Dynamics Reveals an All-or-None Inflammasome Signaling Response. Cell reports 8, 974-982 (2014).

44. Cho, J.S., et al. Neutrophil-derived IL-1 beta is sufficient for abscess formation in immunity against Staphylococcus aureus in mice. PLoS pathogens 8, e1003047 (2012).

45. Seo, S.U., et al. Distinct Commensals Induce Interleukin-1beta via NLRP3 Inflammasome in Inflammatory Monocytes to Promote Intestinal Inflammation in Response to Injury. Immunity 42, 744-755 (2015).

46. Chtanova, T., et al. Dynamics of neutrophil migration in lymph nodes during infection. Immunity 29, 487-496 (2008).

47. Gaya, M., et al. Host response. Inflammation-induced disruption of SCS macrophages impairs B cell responses to secondary infection. Science 347, 667-672 (2015).

48. Lammermann, T., et al. Neutrophil swarms require LTB4 and integrins at sites of cell death in vivo. Nature 498, 371-375 (2013).

49. McDonald, B., et al. Intravascular danger signals guide neutrophils to sites of sterile inflammation. Science 330, 362-366 (2010).

50. Lotze, M.T., et al. The grateful dead: damage-associated molecular pattern molecules and reduction/oxidation regulate immunity. Immunological reviews 220, 60-81 (2007).

51. Zhang, Q., et al. Circulating mitochondrial DAMPs cause inflammatory responses to injury. Nature 464, 104-107 (2010).

52. Amaral, F.A., et al. NLRP3 inflammasome-mediated neutrophil recruitment and hypernociception depend on leukotriene $\mathrm{B}(4)$ in a murine model of gout. Arthritis and rheumatism 64, 474-484 (2012).

53. Kumar, V., et al. Global lymphoid tissue remodeling during a viral infection is orchestrated by a B cell-lymphotoxin-dependent pathway. Blood 115, 4725-4733 (2010).

54. Dostert, C., Ludigs, K. \& Guarda, G. Innate and adaptive effects of inflammasomes on T cell responses. Current opinion in immunology 25, 359-365 (2013).

55. Dixit, V.D. Nlrp3 inflammasome activation in type 2 diabetes: is it clinically relevant? Diabetes 62, 22-24 (2013).

56. Yang, M., Hearnden, C.H., Oleszycka, E. \& Lavelle, E.C. NLRP3 inflammasome activation and cytotoxicity induced by particulate adjuvants. Methods in molecular biology 1040, 41-63 (2013).

57. O'Donnell, H., et al. Toll-like receptor and inflammasome signals converge to amplify the innate bactericidal capacity of T helper 1 cells. Immunity 40, 213-224 (2014).

58. Dunne, A., et al. Inflammasome activation by adenylate cyclase toxin directs Th17 responses and protection against Bordetella pertussis. J Immunol 185, 1711-1719 (2010).

59. Ben-Sasson, S.Z., et al. IL-1 acts directly on CD4 T cells to enhance their antigendriven expansion and differentiation. Proceedings of the National Academy of Sciences of the United States of America 106, 7119-7124 (2009). 
60. Chen, Q., Sen, G. \& Snapper, C.M. Endogenous IL-1R1 signaling is critical for cognate CD4+ T cell help for induction of in vivo type 1 and type 2

antipolysaccharide and antiprotein Ig isotype responses to intact Streptococcus pneumoniae, but not to a soluble pneumococcal conjugate vaccine. J Immunol 177, 6044-6051 (2006). 


\section{Figure legends}

Figure 1. MVA infection induces inflammasome activation, speck formation and rapid loss of SCS macrophages in draining lymph nodes. (a) Detection of caspase-1 activity (FLICA- $1^{+}$staining) in wild-type (WT) and Caspase-1 deficient $\left(\mathrm{Casp}^{-/}\right)$bone marrow derived macrophages (BMDM), $6 \mathrm{~h}$ after infection with MVA (multiplicity of infection, MOI). (b) IL-1 $\beta$ release in WT BMDM cultured with MVA or LPS and ATP (black bars), is inhibited by addition (white bars) of caspase-1 inhibitor z-YVAD-FMK. (c) Three consecutive images ( 2 min intervals) from time-lapse microscopy of BMDM (cell outlined by dashed line) expressing ASC-GFP (green), showing ASC-GFP protein redistribution leading to speck formation induced $6 \mathrm{~h}$ after infection with MVA $(\mathrm{MOI}=25)($ Scale bar: $10 \mu \mathrm{m})$, representative example from more than 10 independent experiments. (d) Detection of ASC specks in cultures of WT BMDM expressing ASC-GFP, $15 \mathrm{~h}$ after treatment with MVA or LPS + ATP. (e) Percentage of dying cells (propidium iodide stain ${ }^{+}$) in WT or Caspl $1^{-/}$ BMDM cultures detected $6 \mathrm{~h}$ after MVA infection by flow cytometry. (f) Immunofluorescent staining of $\mathrm{CD}_{169^{+}}$antigen in popliteal lymph nodes from WT mice up to $18 \mathrm{~h}$ after s.c. injection of $5 \times 10^{6}$ pfu MVA, showing loss of SCS-macrophages (SCS-M) from subcapsular sinus regions of lymph nodes (Scale bar: $100 \mu \mathrm{m}$, representative of four experiments, $n=3$

mice per group). (g) Flow cytometry detection of numbers of lymph node SCS-M, Medullary macrophage (Med-M), macrophage and dendritic cell (DC) subsets in WT mice after injection of MVA (Representative of more than six experiments, $n=6$ mice per time point). Data (a, $\mathrm{b}$, e) are representative of more than three experiments performed in triplicates. All charts plot mean \pm s.e.m. ${ }^{* * P} P<0.01,{ }^{* * *} P<0.001$ compared to WT or vehicle using (d) one-way analysis of variance (ANOVA) followed by Tukey's post hoc test for multiple comparison or $(e, g) S t u d e n t ' s ~ t$-test. 
Figure 2. Dynamic imaging of inflammasome activation in ASC-GFP retrogenic mice reveals rapid kinetics of cellular inflammasome signaling. (a) Schematic of chimeras generated by transfer of $\mathrm{Rag}^{-/-}$bone marrow derived haematopoietic stem cells (BM-HSC), transduced to express ASC-GFP protein, into irradiated recipient mice, in combination with WT or Casp1 $^{-/}$BM cells. (b) Intravital microscopy images of popliteal lymph node in ASCGFP BM-chimeras, showing GFP (green) expression in cells localised to the lymph node collagen capsule (white, second harmonics generation, SHG). Dashed line indicates lymph node edge (Scale bar: $30 \mu \mathrm{m})$. (c) Intravital view of ASC-GFP BM-chimeric mouse lymph node following in vivo staining with a CD169-specific antibody, showing $\mathrm{GFP}^{+}$cell and CD169 colocalization in the subcapsular sinus region (Scale bar: $30 \mu \mathrm{m}$ ). Intravital images of ASC-GFP BM-chimeric mouse lymph node over a time $(t)$ interval of $1 \mathrm{~h}$, showing multiple ASC speck formation events (arrows) occurring following injection with (d) MVA but not (e) PBS injection. Dashed lines indicate lymph node edge and delineate individual ASC-GFP expressing cells (Scale bar: $30 \mu \mathrm{m}$ ). (f) Intravital images of ASC speck formation in a lymph node macrophage $3 \mathrm{~h}$ after local infection with MVA $(t=$ relative to start of ASC-GFP redistribution during speck formation, scale bar: $10 \mu \mathrm{m})$. (g) Kinetics of speck formation in vivo detected by redistribution of ASC-GFP protein (change in mean GFP intensity) from cell cytoplasm (clear circles) to the speck region (green circles) within a single lymph node macrophage on local MVA infection. Data shown (b-f) are representative of 36 individual mice and $(\mathrm{g})$ representative of more than 40 independent events.

Figure 3. Subcapsular macrophages provide a spatiotemporally defined inflammasome response to local viral infection. (a) Intravital tracking of ASC speck formation and its kinetics in popliteal lymph node following local MVA infection. Each horizontal line 
represents an individual ASC-GFP ${ }^{+}$cell and each square represents a 10 min time period. (b) Quantification of inflammasome activity in draining lymph node $0-12 \mathrm{~h}$ following local injection of PBS (triangles) or MVA (circles). Axes show the number of formed (left axis, green circles) and actively forming (right axis, red circles) ASC specks detected by intravital microscopy, acquired over more than 125 movies where each dot represents the mean \pm s.e.m number of events detected at each experimental time point $(n=8-33$ movies acquired from at least three mice analyzed over each time point shown). Data fit to a Log Gaussian curve is presented. (c) Plot showing the percentage of ASC-GFP specks detected at distances up to $100 \mu \mathrm{m}$ relative to the position of the lymph node capsule $(0 \mu \mathrm{m}$; identified by second harmonics signal generation by collagen fibers). Data shows mean \pm s.e.m percentage of 35 speck events detected within 11 imaging fields (five independent experiments) each acquired with up to $100 \mu \mathrm{m}$ imaging depths. Data shown (a, c) are compiled of more than 40 independent events.

Figure 4. ASC aggregation precedes rapid cell death releasing long-lived extracellular ASC specks in vivo. (a) Time-lapse microscopy (hh:mm post infection) of WT BMDM expressing ASC-mCherry protein (top panel), showing nuclear staining with SytoxBlue occurs rapidly following speck formation, on infection with MVA-GFP (merged, middle panel). Transmitted light images confirm cell death (lower panel) (Scale bar: $10 \mu \mathrm{m}$ ). Representative images from more than five independent experiments. (b) Following infection with MVA-GFP, changes in BMDM cytoplasmic GFP intensity (left axis) and nuclear SytoxBlue (right axis), show rapid loss of cell integrity relative to time (min) of full ASC speck formation (red line). Plot shows mean fluorescence intensity values $( \pm$ s.e.m) of 25 cellular events as shown in (a). (c) In situ staining of lymph node macrophages with Hoechst 33342 in ASC-GFP BM-chimeric mice shows MVA-induced ASC formation is associated 
with rapid nuclear condensation (Scale bar: $10 \mu \mathrm{m}$ ). Dotted lines outline detectable cell cytoplasm and nuclear regions. (d) Intravital imaging of ASC-GFP BM-chimeric mouse lymph nodes following MVA treatment $(6 \mathrm{~h})$ show locally injected ASC-specific PE antibody colocalize with formed ASC-GFP specks (Scale bar: $40 \mu \mathrm{m}$, inset images $20 \mu \mathrm{m}$ ). (e) Intravital tracking of formed ASC specks induced by MVA infection show they are long lasting entities with variable fates. Horizontal lines in summary table represent an individual formed speck tracked in vivo, where each square describes changes in specks observed over 10-minute periods. (f) Representative intravital images of speck fates (Scale bar: $10 \mu \mathrm{m}) .(\mathbf{g})$ In situ staining of lymph node resident cells with Hoechst 33342, followed by intravital imaging reveals ASC speck formation (green) induces rapid local cell recruitment and clustering in lymph nodes of ASC-GFP BM-chimeric mice during MVA infection (scale bar: $10 \mu \mathrm{m})$. Examples shown are representative of three (d) or five $(\mathrm{c}, \mathrm{g})$ independent experiments, (e,f) are representative of more than 80 independent events $(n=28$ mice).

Figure 5. Inflammasome activation regulates the magnitude of the inflammatory innate infiltrate into draining lymph nodes. (a) Whole lymph node tissue chemokine profiling performed $4 \mathrm{~h}$ after MVA or PBS injection in WT, $\operatorname{Caspl}^{-/-}$and $I l 1 r 1^{-/-}$mice, where values shown are detected protein levels normalised to total lymph node protein content $(n=3)$. (bd) Absolute numbers of $\mathrm{CD} 11 \mathrm{~b}^{\mathrm{hi}} \mathrm{Ly} 6 \mathrm{G}^{+}$neutrophils, $\mathrm{Ly} 6 \mathrm{C}^{\mathrm{hi}} \mathrm{CD} 11 \mathrm{~b}^{+}$monocytes and NK cells detected in lymph nodes by flow cytometry, up to $18 \mathrm{~h}$ after s.c. MVA infection in WT (black filed circles), $\mathrm{Caspl}^{-/-}$(clear circles) and $\mathrm{IlIrI}^{-/-}$(grey filed circles) mice. Plots show combined data from more than six independent experiments. (e,f) Absolute numbers of neutrophils (e) and monocytes (f) detected in lymph nodes of BM chimeric mice (Illr $1^{-/-}>$ $\mathrm{WT}$, red bars; WT $>I l 1 r 1^{-/-}$, grey bars; control group WT $>\mathrm{WT}$, black bars) by flow cytometry, $4 \mathrm{~h}$ (left plot) and $18 \mathrm{~h}$ (right plot) after MVA infection or in PBS controls. Data 
shown ( $n=5$ animals per group) is representative of two independent experiments. All charts

plot mean \pm s.e.m. ${ }^{* *} P<0.01,{ }^{* * *} P<0.001$ compared to WT or vehicle using one-way analysis of variance (ANOVA) with Tukey's multiple comparison test (b-f) or Mann-Whitney $\mathrm{U}$ test $(\mathrm{a}) * P<0.001$

Figure 6. Inflammasome signals recruit circulating antigen-specific $T$ cells to the draining lymph node. (a) Flow cytometry pentamer (H2K $-\mathrm{TSY})$ staining for detection of MVA vaccinia B8R peptide-specific T cells in draining lymph nodes 6 days post-infection, summarized as (b) percentages (of total $\mathrm{CD}^{+} \mathrm{T}$ cells) and (c) absolute cell numbers in WT, $\operatorname{Caspl}^{-/-}$and $I l 1 r 1^{-/-}$mice. Representative plots and experiments are shown ( $n=5$ mice). (d) Absolute numbers of $\mathrm{CD}^{+}$and (e) $\mathrm{CD}^{+} \mathrm{T}$ cells detected in lymph nodes by flow cytometry, up to $18 \mathrm{~h}$ after s.c. MVA infection. (f) Schematic representation of the experimental approach, involving transfer of cyan fluorescent protein (CFP) or CD45.1 $1^{+} \mathrm{T}$ cells into CD45.2 $2^{+}$recipient mice $(1: 2$ ratio), followed by draining lymph node (dLN) analysis. Combined data from three independent experiments $(n=8$ mice). (g,h) Representative plots of the composition of $\mathrm{H}_{2} \mathrm{~K}^{\mathrm{b}}$-TSY-specific $\mathrm{CD}^{+} \mathrm{T}$ in draining lymph nodes, 6 days after MVA infection, showing transferred $\mathrm{CFP}^{+}$(blue) and $\mathrm{CD} 45.1^{+}$(red) $\mathrm{T}$ cells, where percentages as a proportion of total pentamer-specific $\mathrm{T}$ cells are represented in the pie charts. All charts plot mean and error bars represent the s.e.m. ${ }^{* *} P<0.01,{ }^{* * *} P<0.001$ compared to WT, using one-way ANOVA with Tukey’s multiple comparison test. 


\section{Online methods}

Mice. Wild type (WT) C57B1/6 mice were purchased from Charles River France. Caspase-1

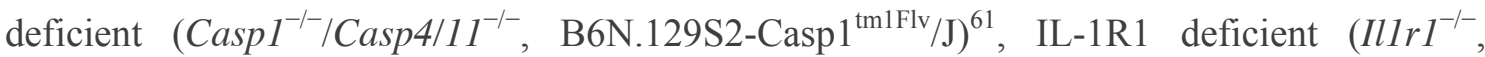

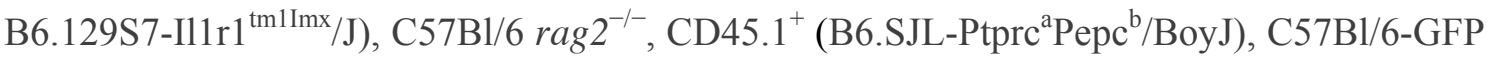
(GFP expressed under the ubiquitin promoter) and C57Bl/6-CFP (CFP expressed under the actin promoter) mice were bred in our animal facility under specific pathogen-free conditions and littermates were randomly assigned to experimental treatment groups. Experimental protocols were ethically approved by the Institutional Committees on Animal Welfare at Institut Pasteur and conducted in strict accordance with French guidelines for animal care and use.

\section{Treatments \& Reagents}

Recombinant MVA-B (expressing full-length HIV Gag, fused to 3 Pol and 2 Nef fragments) ${ }^{62}$ was provided by the Agence Nationale de Recherche sur le Sida (ANRS), and a recombinant MVA-B strain expressing enhanced GFP was provided by Transgene Laboratories (Strasbourg, France). Mice were injected subcutaneously (s.c.) in the footpad with $5 \times 10^{6} \mathrm{pfu}$ of MVA. In vivo labeling of $\mathrm{CD} 169^{+}$cells was performed by s.c. injection of $0.5 \mu \mathrm{g}$ PEconjugated anti-CD169 antibody (Biolegend, clone 3D6.112) approximately 1 hour before imaging. In vivo staining of extracellular ASC specks was performed by injection of $0.5 \mu \mathrm{g}$ AF555-conjugated anti-ASC antibody (Bioss Antibodies, bs-6741R-A555) 6 hours after MVA or PBS injection, and imaging commenced within 30 minutes. Whole lymph node labeling with Hoechst 33342 (33342 trihydrochloride, trihydrate, Invitrogen) was performed by s.c. injection of $10 \mu \mathrm{L}$ of a $0.1 \mu \mathrm{M}$ solution prepared in PBS and imaging commenced within 30 minutes. The investigators were not blinded during experiments or data analysis. In vivo blockade of CD62L, was performed by i.v. injection of $100 \mu \mathrm{g}$ blocking antibody 
(ebioscience, MEL-14) 6 hours before MVA injection and a further 24 hours after MVA injection. WT mice were treated with a neutralizing IL-1 $\beta$ antibody (R\&D systems, AF-401NA), by administration of $100 \mu \mathrm{g}$ i.p. 1 hour before T cell transfer or MVA injection.

\section{Flow cytometry}

To detect caspase activity, macrophages $\left(0.2 \times 10^{6}\right.$ cells $)$ were seeded in ultra-low attachment 24-well plates (Corning) overnight and then stimulated with MVA expressing a fluorescent protein to monitor infection, at 1, 5, 25 or 50 multiplicity of infection (MOI) for 6-10 hours. Cells were gently harvested and stained with a FLICA-1 FITC (caspase-1 probe FAMYVAD-FMK) and FLICA-3/7 660 (caspases-3/7 FAM-DEVD-FMK) kits according to the manufacturers' instructions (Immunochemistry Technologies). Control cultures were treated with $1 \mu \mathrm{M}$ Staurosporine (STS) (Sigma) to induce caspase-3 activity. To detect tissue infection by MVA and inflammatory cell recruitment, popliteal lymph nodes were teased apart using forceps and digested in RPMI 1640 medium containing $1 \mathrm{mg} / \mathrm{ml}$ collagenase D and $50 \mathrm{ng} / \mathrm{ml}$ DNase (Sigma) for 15 minutes at $37^{\circ} \mathrm{C}$, and then passed through a $70-\mu \mathrm{m}$ cell strainer. Virally expressed GFP was detectable directly by flow cytometry. Cell-surface staining was performed using the following antibodies at a dilution of 1:200 $(0.5 \mu \mathrm{g} / \mathrm{mL})$ unless specified, in the presence of blocking anti-CD16/32 (eBioscience, clone 93) and following exclusion of doublets and dead cells (Live/dead fixable dyes, Invitrogen): anti-CD3 (17A2), anti-NK.1.1 (PK136), anti-CD8 (53-6.7), anti-CD19 (1D3), anti-CD169 (3D6.112/ MOMA-1, AbD Serotec,), anti-CD11b (M1/70, BD), anti-F4/80 (BM8), anti-CD45.1 (A20), anti-CD45.2 (104), anti-CD11c (N418), (1:500) anti-I-A/E (M5/114.15.2), (1:400) anti-Ly-6G (1A8), anti-Ly-6C (AL-21), all from Biolegend or other suppliers where indicated. Cell subset analysis was performed based on the following phenotypic gating definitions: $\mathrm{CD}^{-} \mathrm{CD}^{-} 1 \mathrm{~b}^{+}$ $\mathrm{CD}_{169^{+}} \mathrm{F} / 80^{-}$for SCS-M, CD3 ${ }^{-} \mathrm{CD} 1 \mathrm{~b}^{+} \mathrm{CD} 169^{+} \mathrm{F} 4 / 80^{+}$for Med-M, $\mathrm{CD}^{-} \mathrm{Ly}^{-} \mathrm{G}^{-} \mathrm{CD}^{-} 6^{-}$ 
$\mathrm{CD}_{11 \mathrm{~b}^{+}}$for macrophages, $\mathrm{CD}^{-} \mathrm{I}-\mathrm{A} / \mathrm{E}^{\mathrm{hi}} \mathrm{CD} 11 \mathrm{c}^{+}$for $\mathrm{DCs}, \mathrm{CD}^{-} \mathrm{Ly} 6 \mathrm{G}^{-} \mathrm{Ly} \mathrm{C}^{\mathrm{hi}} \mathrm{CD} 11 \mathrm{~b}^{+}$for inflammatory monocytes, $\mathrm{CD} 11 \mathrm{~b}^{\mathrm{hi}} \mathrm{Ly}_{6 \mathrm{G}}{ }^{+}$for neutrophils and $\mathrm{CD} 11 \mathrm{~b}^{+} \mathrm{NK} 1.1^{+}$for NKs cells. Absolute cell numbers were quantified using AccuCheck bead microspheres (Life Technologies) during flow acquisition. Intracellular staining was performed using the Cytofix/Cytoperm kit (BD Bioscience) using APC-conjugated anti-GranzymeB (GB11, BD biosciences) and FITC-conjugated anti-IFN $\gamma$ (XMG1.2, ebioscience). Analyses were performed using an LSRII Cytometer (BD Biosciences) and analyzed using FlowJo software version 10.6 (Tree Star). Cell death induced by MVA was detected in BMDM cultures infected with MVA-GFP by counterstaining with $2.5 \mu \mathrm{g} / \mathrm{mL}$ propidium iodide (Sigma).

\section{ELISA}

For ELISA detection of IL-1 $\beta$ in cell free supernatants, BMDM were cultured overnight with MVA (multiplicity of infection (MOI): 1-50) or $1 \mu \mathrm{g} / \mathrm{mL}$ LPS (serotype 026:B6, Sigma) in the presence or absence of $75 \mu \mathrm{M}$ caspase-1 inhibitor Z-YVAD-FMK (R\&D Systems). The next day LPS treated cells were pulsed with 5mM adenosine 5'-triphosphate disodium salt hydrate ATP for 1 hour. Supernatants were harvested and analyzed using IL-1 $\beta$ DuoSet ELISA kit according to the manufacturer's instructions (R\&D Systems).

\section{Inflammasome ASC probe generation}

Constructs ASC-GFP (enhanced GFP) or ASC-mCherry (monomeric cherry) ${ }^{63}$ encoding the full sequence of ASC, with fluorescent protein tagged onto the 5' sequence end, were cloned into a murine stem cell viral (MSCV) vector using standard techniques. Retrovirus was generated by co-transfection of ASC-MSCV $(30 \mu \mathrm{g})$ and pCI-Eco $(10 \mu \mathrm{g})$ plasmid DNA into HEK 293 T cells using Lipofectamine 2000 transfection reagent $(60 \mu \mathrm{L})$ (Life Technologies), in Opti-MEM media (Gibco), which was quenched after 8 hours with Dulbecco's Modified 
Eagle Medium (DMEM) containing 10\% FBS, 1\% Penicillin/ streptomycin. After overnight incubation at $37^{\circ} \mathrm{C}$, culture media was replaced with either complete DMEM or Iscove's Modified Dulbecco's Medium (IMDM), if the retrovirus was to be used for transduction of BMDM or hematopoietic stem cells (HSCs), respectively. Retrovirus containing supernatant was harvested from $293 \mathrm{~T}$ cell cultures during the next 2 days, filtered $(0.45 \mu \mathrm{M})$ and used to perform sequential rounds of BMDM and HSC transduction as described below.

\section{Bone marrow derived macrophage generation and transduction}

Murine bone marrow derived macrophages (BMDM) from WT or Caspl $^{-/-}$mice were differentiated from bone marrow $\left(1-1.5 \times 10^{6}\right.$ cells seeded in $9.5 \mathrm{~cm}^{2}$ wells $)$ by culture in DMEM, 10\% FBS, 1\% Penicillin/ Streptomycin, 1\% Hepes (4-(2-hydroxyethyl)-1piperazineethanesulfonic acid) (all from Gibco) with 20ng/mL (macrophage-colony stimulating factor) M-CSF (eBioscience) or 20\% L929 supernatant, and used at day 7. BMDM were stably transduced to express ASC-GFP/ mCherry by adding retroviralconditioned medium to macrophage cultures on days 3 and 4 during their differentiation. Retroviral supernatant was supplemented with fresh DMEM, 10\% FBS, $14 \mu \mathrm{g} / \mathrm{mL}$ Polybrene (Sigma), 1\% Hepes and 20ng/mL M-CSF (eBioscience) was used to replace media on BMDM cultures and incubated at $37^{\circ} \mathrm{C}$ overnight. This was repeated the following day, where after overnight culture, retroviral media was replaced with fresh culture media. Transduction efficiencies of $>70 \%$ were routinely achieved.

\section{Time-lapse videomicroscopy}

Macrophages transduced to express ASC-GFP or ASC-mCherry $\left(0.2 \times 10^{6}\right.$ cells $)$ were seeded in $35 \mathrm{~mm}$ FluoroDish cell culture dishes (World Precision Instruments) in phenol-red free RPMI supplemented with 10\% FBS, 1\% Penicillin/ Streptomycin and 10ng/mL M-CSF for 6 
hours, with additional $1 \mu \mathrm{g} / \mathrm{mL}$ LPS where indicated. Immediately prior to imaging, cell media was replaced with media containing MVA, MVA-GFP or 5mM ATP and supplemented with $0.1 \mu \mathrm{M}$ SytoxBlue (Invitrogen). Time-lapse microscopy was performed using a DMI6000B inverted microscope (Leica Microsystems) equipped with heated chamber for temperature, humidity, and $\mathrm{CO}_{2}$ control, with a 10X/ 0.75 NA dry objective (Olympus) and a CoolSNAP HQ2 Roper camera (Photometrics) for 15 hours after induction. Videos were analyzed using ImageJ or FiJi softwares (National Institutes of Health, http://rsb.info.nih.gov/ij/).

\section{Bone marrow chimeras}

To generate mixed BM chimeric mice for intravital imaging of inflammasome activity, lethally irradiated mice were reconstituted with BM-derived HSCs, retrovirally transduced to stably express ASC-GFP protein. Donor HSCs used for reconstitution were negatively selected (Miltenyi, Lineage depletion kit) from C57Bl/6 $\mathrm{Rag}^{-/-} \mathrm{BM}$ (to achieve myeloid-

restricted probe expression) and cultured overnight $\left(2-3 \times 10^{6}\right.$ cells $/ 9.5 \mathrm{~cm}^{2}$ well $)$ in complete Iscove's Modified Dulbecco's Medium (IMDM), supplemented with 10\% FBS, 1\% Hepes, 0.1\% 2-mercaptoethanol, 20ng/mL Stem cell factor/ c-Kit ligand (SCF) and $10 \mathrm{ng} / \mathrm{mL}$ Thrombopoietin (TPO). After culture overnight, HSC cell cultures were resuspended in ASCGFP retroviral supernatant (supplemented with 10\% FBS, 1\% Hepes, $0.1 \%$ 2mercaptoethanol, 20ng/mL SCF, 10ng/mL TPO, 10 $\mu \mathrm{g} / \mathrm{mL}$ Polybrene) and spun-transduced at $1000 \mathrm{~g}, 32^{\circ} \mathrm{C}$ for 90 minutes. Fresh complete IMDM media was added after a further 4-6 hours incubation at $37^{\circ} \mathrm{C}$. This was repeated the following day, with the exception that 4-6 hours after spin-transduction, HSCs were harvested, washed in fresh media, and seeded onto pre-prepared $10 \mathrm{~cm}^{2}$ dishes of OP-9 feeder cells (ATCC) ${ }^{64}$ in complete IMDM media. Cultures were harvested after a further 36-48 hours and $\mathrm{ASC}^{-G_{F P}}{ }^{+}$cells sorted on a FACSAria II (Becton Dickenson). Recipient WT mice were lethally irradiated by x-ray ( 8.5 
grays, X-Xstrahl RS320), 4-6 hours before injection of $>0.2 \times 10^{6}$ ASC-GFP HSCs per mouse. Sorted ASC-GFP ${ }^{+}$HSCs were injected in combination with either WT or Caspl $^{-/-}$BM HSCs $^{-1}$ to achieve $90 \%-10 \%$ chimerism. This served to restrict caspase-1 functional inflammasome signaling only to probe expressing cells when used for experiments to examine inflammasome effector functions, and also enabled a discrete distribution of $\mathrm{ASC}-\mathrm{GFP}^{+}$cells for easier single cell tracking in vivo. Chimeric mice had a normal lymphoid architecture as mixed chimeras were generated partially with replete BM to reconstitute the lymphoid compartment. Recipient mice were administered with sulfamethoxazole and trimethoprim supplemented water (Roche) for the following 2 weeks, and were used after $>8$ weeks following BM reconstitution, which was verified by histological and flow cytometry examination of blood and lymphoid tissues. Bone marrow chimeric mice to study IL-1 sensing were prepared by transferring freshly isolated bone marrow cells $\left(50-100 \times 10^{6}\right.$ cells $)$ derived from CD $45.1^{+}$ $I l l r 1^{-/-}$mice into lethally irradiated CD $45.2^{+}$WT recipients. Reconstitution of typically $>95 \%$ was confirmed by blood and lymphoid tissue congenic marker expression.

\section{Whole tissue chemokine profiling}

Multianalyte profiling of whole popliteal lymph node tissue homogenates was made by RodentMAP analysis (MyriadRBM). Popliteal lymph node specimens were prepared 4 hours after MVA or PBS injection by pooling 4 excised lymph nodes and homogenising tissues using a Potter-Elvehjem homogenizer in $350 \mu$ of lysis buffer $(50 \mathrm{mM}$ Tris-HCL pH 7.4, 2 mM EDTA) containing aprotinin, antipain, leupeptin, and pepstatin A (all at $1 \mu \mathrm{g} / \mathrm{mL}$ ) and 2mM PMSF (phenylmethylsulfonyl fluoride), at $4^{\circ} \mathrm{C}$ (Roche Diagnostics). Total protein content of each tissue lysate was quantified (BCA Assay, Pierce, Rockford USA) and used to normalize concentrations of analytes detected within each sample, to the total sample protein content. 


\section{Immunofluorescence histological analysis}

Whole excised lymph nodes were fixed at $4{ }^{\circ} \mathrm{C}$ overnight in paraformaldehyde and progressively dehydrated in sucrose. Tissues were snap frozen in OCT compound (TissueTek; Sakura) and $8 \mu \mathrm{m}$ thick tissue cryosections prepared. Antibody staining of F4/80, CD169 and GFP (Invitrogen, PA1-46326) was performed following blocking in mouse and rat serum, and sections imaged using a confocal microscope (Olympus BX61WI).

\section{Intravital two-photon imaging}

Two-photon imaging of intact popliteal lymph node was performed as previously described ${ }^{65}$,

${ }^{66}$, using an upright microscope (DM6000B, Leica Microsystems) with a 20X/0.95 NA waterdipping objective (Olympus). Excitation was provided by a Chameleon Ultra Ti:Sapphire (Coherent) tuned to $950 \mathrm{~nm}$. The following filter sets were used for imaging second harmonics generation (SHG)/ GFP/ PE, and Hoechst/ GFP: 483/32 BP, 495 LP, 520/35 BP, 562 LP, $607 / 20$ B; 483/32 BP, 495 LP, 520/35 BP. Image fields of $1024 \times 1024$ pixels, with a mean pixel size of $0.5 \mu \mathrm{m}$, z-spacing of $4 \mu \mathrm{m}$ and a volume depth of up to $100 \mu \mathrm{m}$ were recorded. Datasets were processed and analyzed using Imaris (Bitplane) and FiJi softwares.

\section{Antigen-specific $\mathbf{T}$ cell detection}

Antigen-specific T cell responses induced by MVA were detected within spleen and draining popliteal lymph nodes 6 days after infection, using contralateral PBS treated lymph nodes as controls. $\mathrm{CD}^{+} \mathrm{T}$ cells with specificity for the immunodominant vaccinia viral peptide (VVp) B8R 20-27 were detected by pentamer staining (PE-conjugated H2-K ${ }^{\mathrm{b}}$-TSYKFESV, Proimmune) of whole lymphoid cell suspensions, in $\mathrm{CD}^{+} \mathrm{CD}^{-} 9^{-}$and $\mathrm{NK} 1.1^{-}$gated cells by flow cytometry. For adoptive transfer assays to assess recruitment and antigen-specific $\mathrm{T}$ cell 
priming of peripherally recruited $\mathrm{T}$ cells, we purified $\mathrm{CD}^{+} \mathrm{T}$ cells from $\mathrm{C} 57 \mathrm{Bl} / 6-\mathrm{GFP}$ $\left(20 \times 10^{6}\right.$ cells $)$, or $\mathrm{C} 57 \mathrm{Bl} / 6-\mathrm{CFP}$ and $\mathrm{CD} 45.1^{+}$mice $\left(10 \times 10^{6}\right.$ and $20 \times 10^{6}$ cells respectively) and injected cells $10 \times 10^{6}$ and $20 \times 10^{6}$ cells respectively into CD45.2 $2^{+} \mathrm{WT}, \mathrm{Caspl}^{-/}$and $I l 1 r 1^{-/-}$mice, followed by s.c. injection of MVA. Six days later, draining lymph node cell suspensions were analyzed by flow cytometry to detect the proportion of transferred cells within pentamer ${ }^{+} \mathrm{CD}^{+} \mathrm{CD}^{-} 9^{-}$and $\mathrm{NK} 1.1^{-}$populations. To examine the $\mathrm{H} 2-\mathrm{K}^{\mathrm{b}}$ restricted antigen-specific $\mathrm{CD}^{+} \mathrm{T}$ cell response to MVA peptide antigens A8R (189, ITYRFYLI), A3L (270-277, KSYNYMLL), B8R (20-27, TSYKFESV), K3L (6-15, YSLPNAGDVI), and A19L (47-55, VSLDYINTM) (Polypeptide Laboratories), $1 \times 10^{6}$ cells isolated from draining lymph nodes 6 days after MVA injection, were cultured in the presence or absence of $1 \mu \mathrm{g} / \mathrm{mL}$ of each peptide and 1x GolgiPlug (BD bioscience) in complete medium for 4 hours, followed by intra-cellular cytokine staining.

\section{Statistical analyses}

All statistical tests were performed using Prism v6.0b (GraphPad) and all data are represented as mean \pm SEM. Group comparisons were assessed with non-parametric tests. We used Student's $t$ test for two groups comparison or Kruskal-Wallis test for multiple comparisons.

One-way ANOVA test followed by Tukey’s post hoc test was used for multiple comparisons of data with normal distribution and equal variance. All statistical tests were two-tailed with a significance level of 0.05 . ${ }^{*} P<0.05, * * P<0.01, * * * P<0.001$, ns non-significant. No statistical test was used to predetermine sample size and all samples were included in the analysis.

\section{Methods references}

61. Kuida, K., et al. Altered cytokine export and apoptosis in mice deficient in interleukin-1 beta converting enzyme. Science 267, 2000-2003 (1995). 
62. Brandler, S., et al. Preclinical studies of a modified vaccinia virus Ankara-based HIV candidate vaccine: antigen presentation and antiviral effect. Journal of virology 84, 5314-5328 (2010).

63. Stutz, A., Horvath, G.L., Monks, B.G. \& Latz, E. ASC speck formation as a readout for inflammasome activation. Methods in molecular biology 1040, 91-101 (2013).

64. Nakano, T., Kodama, H. \& Honjo, T. Generation of lymphohematopoietic cells from embryonic stem cells in culture. Science 265, 1098-1101 (1994).

65. Garcia, Z., et al. Subcapsular sinus macrophages promote NK cell accumulation and activation in response to lymph-borne viral particles. Blood 120, 4744-4750 (2012).

66. Celli, S., Breart, B. \& Bousso, P. Intravital Two-Photon Imaging of Natural Killer Cells and Dendritic Cells in Lymph Nodes. Methods Mol Biol 415, 119-126 (2008). 


\section{Sagoo et al. Figure 2}

a

Rag2 $^{-/} \quad \begin{gathered}\text { Retroviral } \\ \text { transduction }\end{gathered}$

BM-HSC ASC-GFP

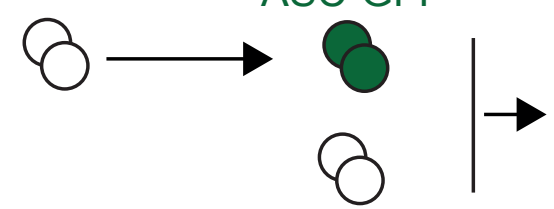

WT or Casp1 ${ }^{-1-} \quad$ Myeloid-specific BM-HSC ASC-GFP expression
Mixed BM chimera

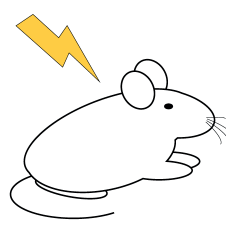

b

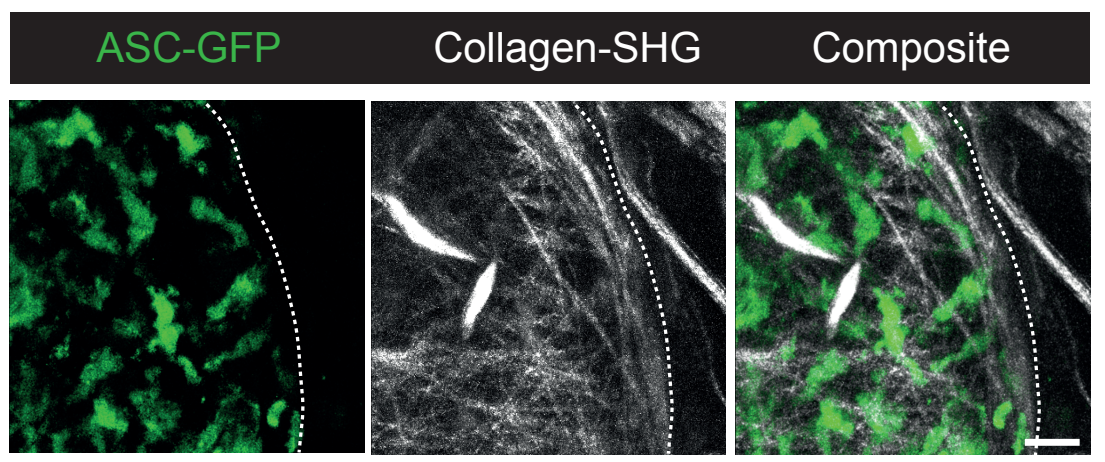

e

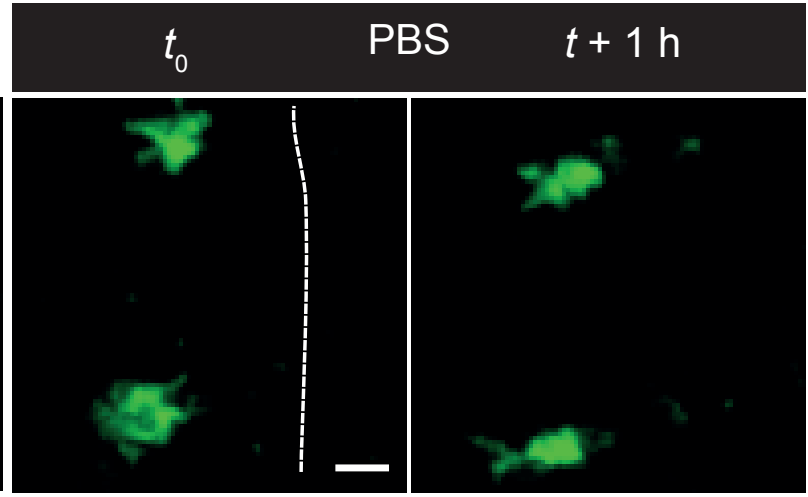

f

C

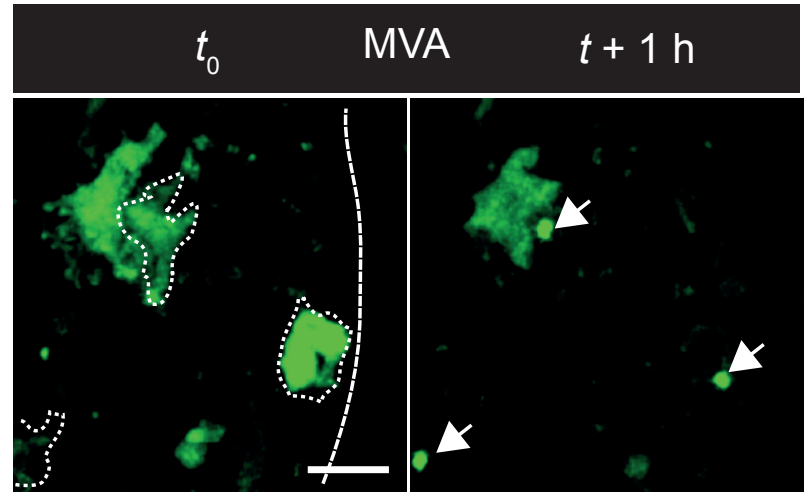

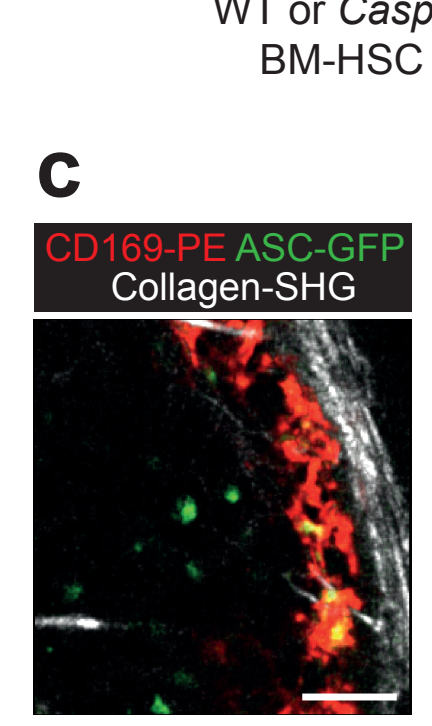
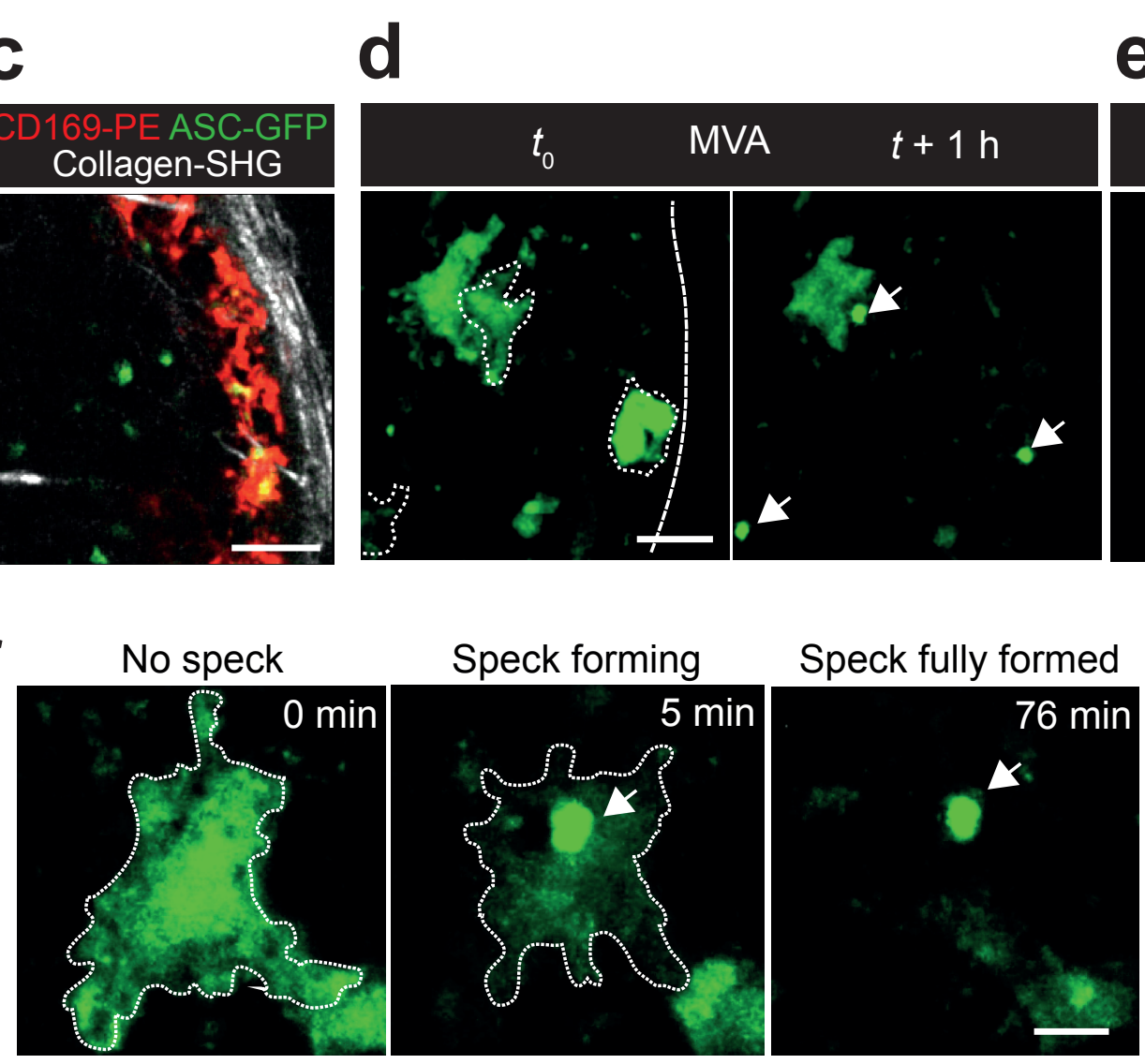

9

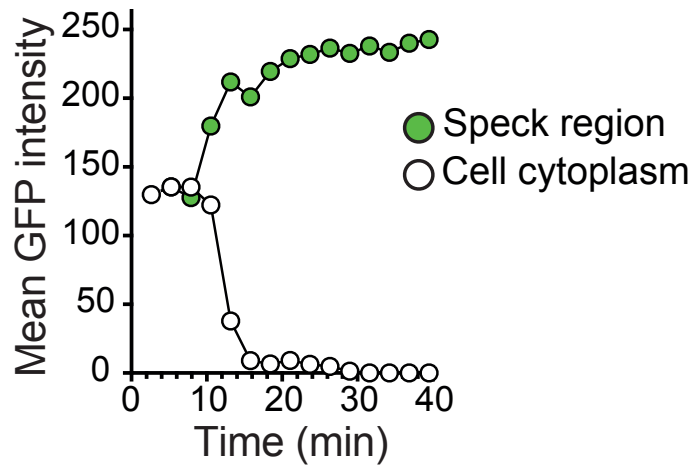




\section{Sagoo et al. Figure 3}

a

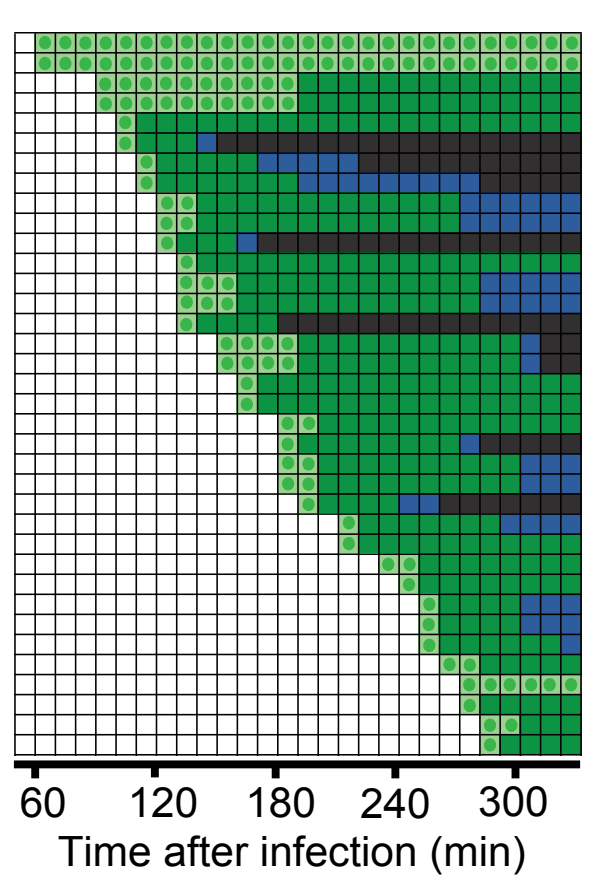

Time after infection (min)

$\square$ No speck

$\square$ Speck partially formed Speck fully formed $\square$ Speck diminishes $\square$ Speck disapears b
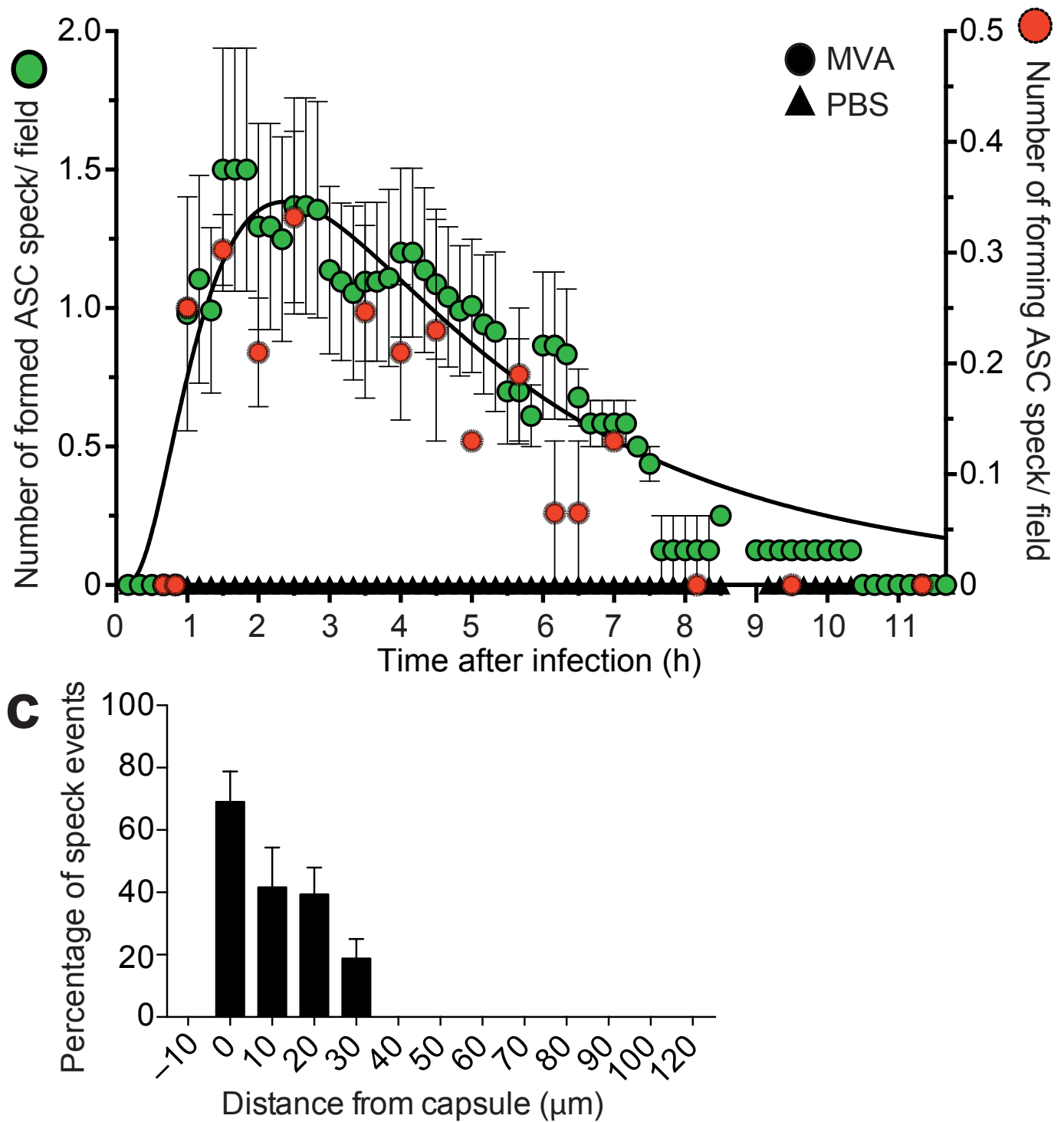
Sagoo et al. Figure 4

a

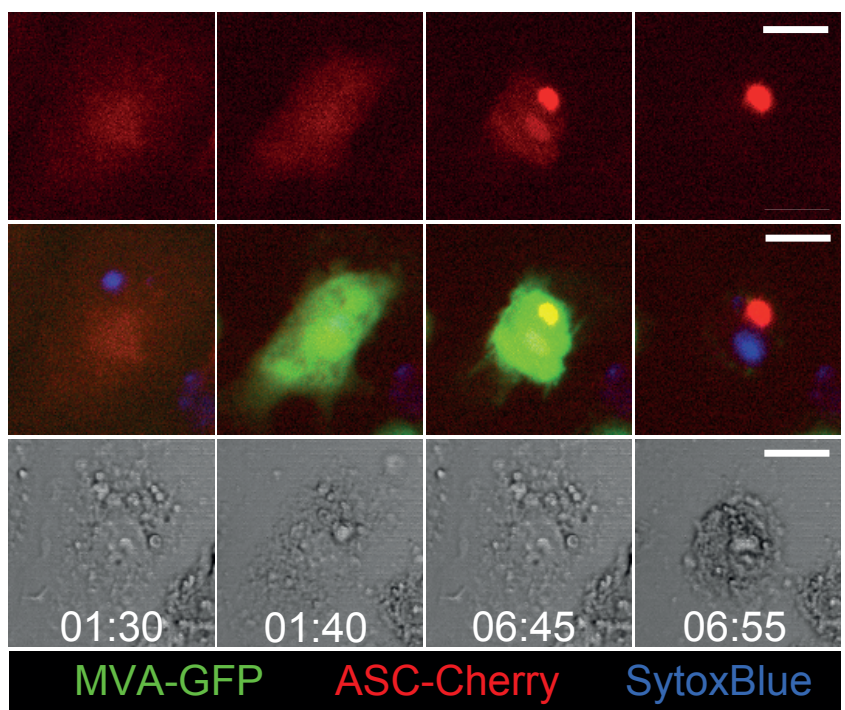

b

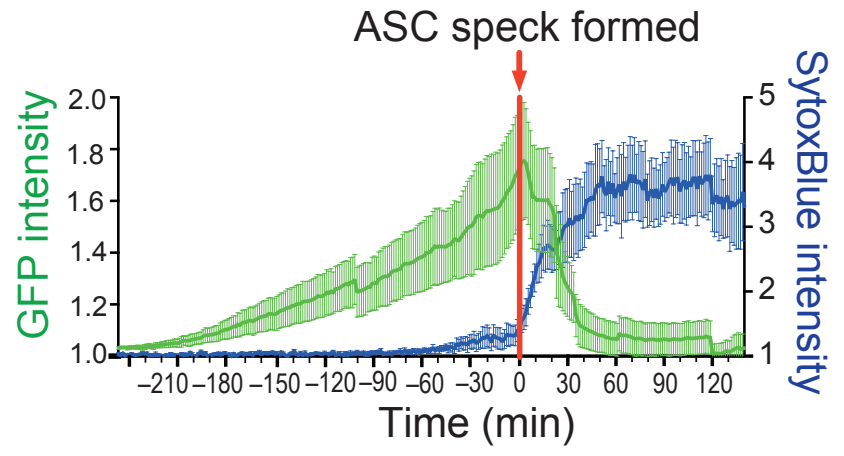

C

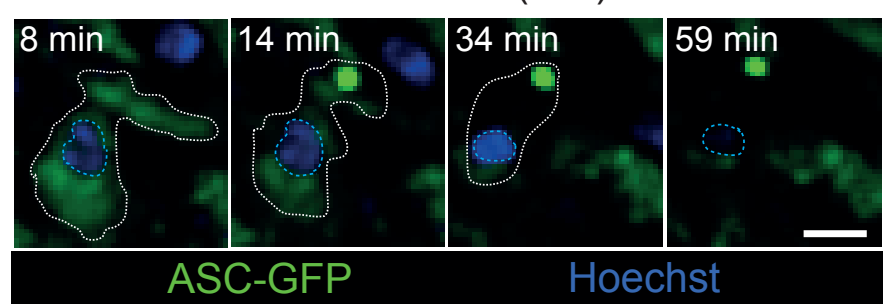

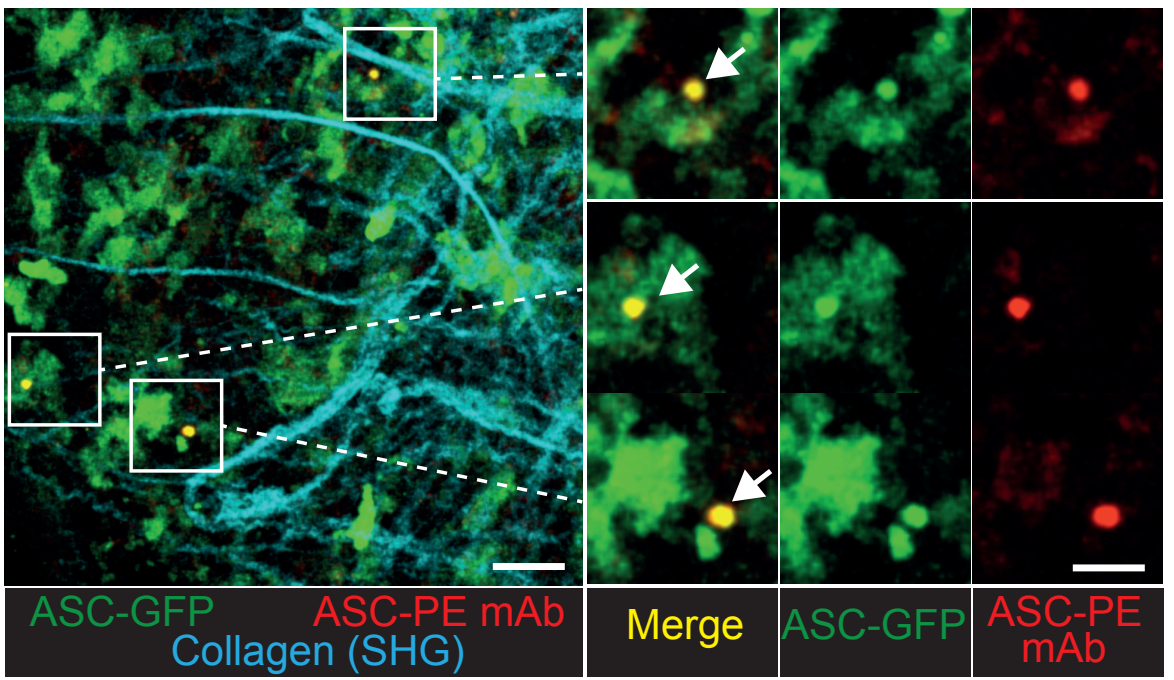

f

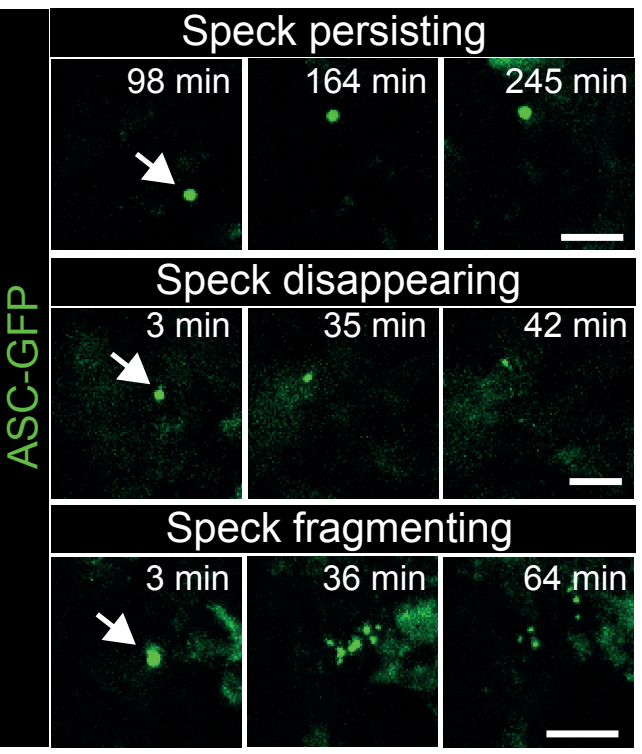

Speck fully formed Speck partially formed $\square$ Speck diminishes Speck disappears Speck fragments

g
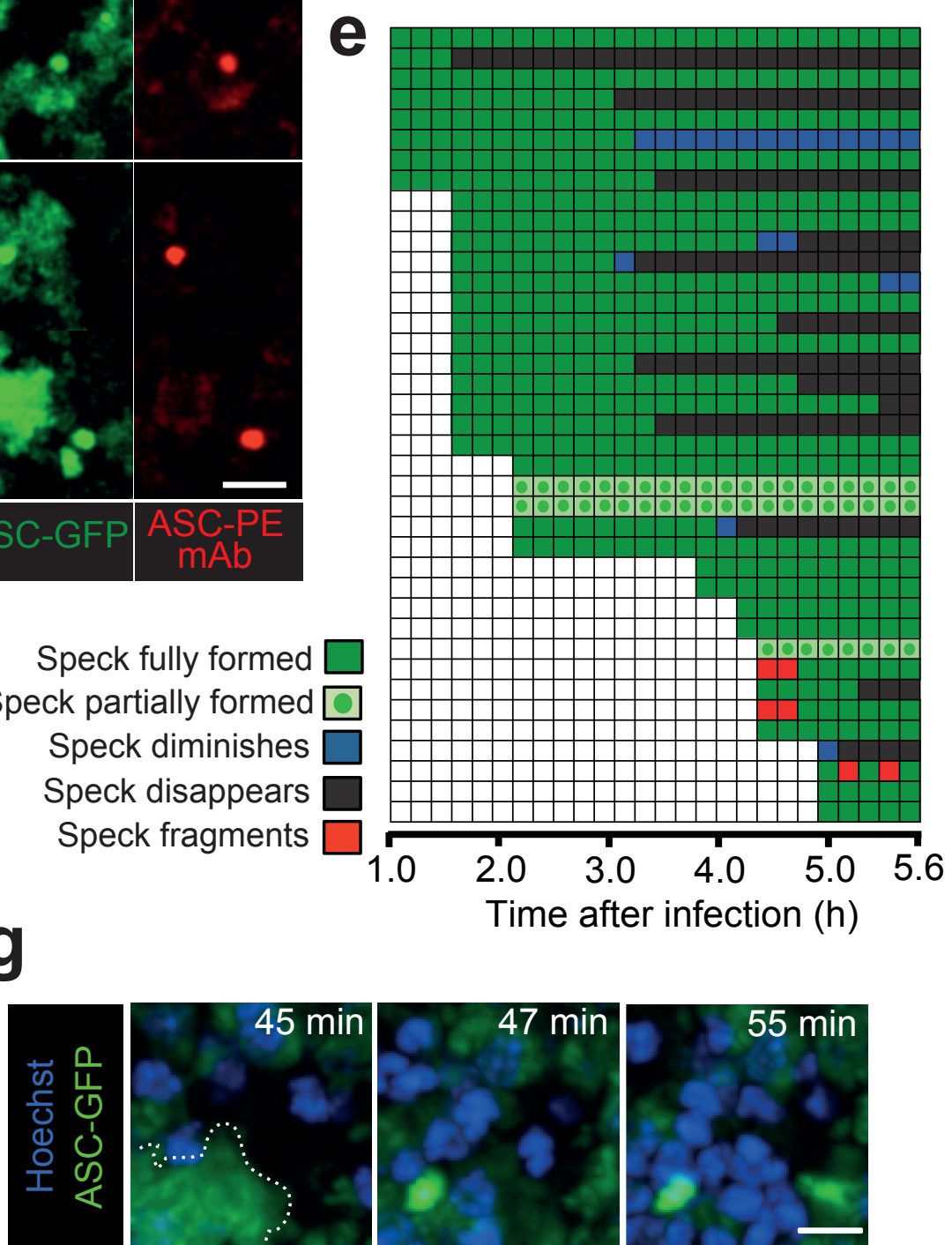


\section{Sagoo et al. Figure 5}

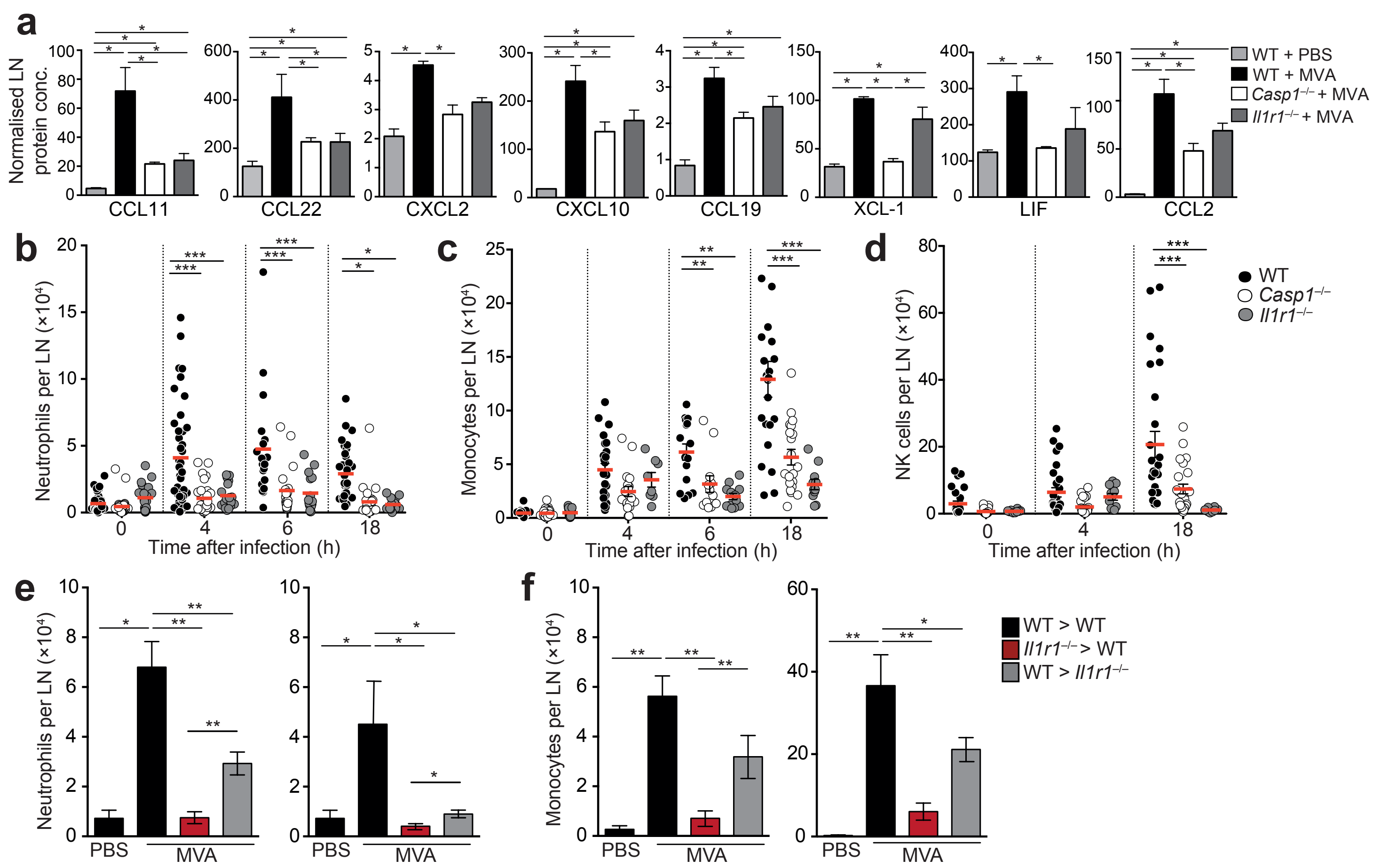


